\title{
Estudio sobre los cementos portland fosfáticos
}

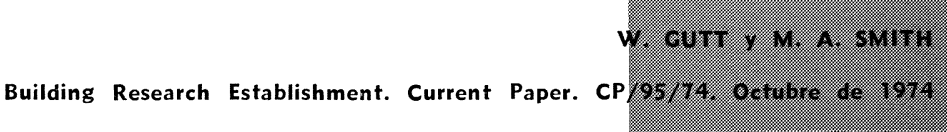

Esta comunicación fue presentada al 6. Simposio Internacional sobre Química del Cemento, celebrado en Moscú en septiembre de 1974.

Los primeros estudios sobre el equilibrio de fases a alta temperatura del sistema $\mathrm{CaO}$ $\mathrm{SiO}_{2}-\mathrm{P}_{2} \mathrm{O}_{5}$ han proporcionado unas bases fundamentales para comprender el papel de los fosfatos en la fabricación del cemento portland a partir de materias primas que contengan fosfatos. También ha servido para poner en evidencia la especial importancia de los fluoruros como mineralizantes en la fabricación de los cementos fosfáticos. Recientemente se ha hecho una evaluación sobre la idoneidad de algunas materias primas fosfatadas de diferentes orígenes para la fabricación de cemento y de estos resultados se ha podido confirmar cuál es el nivel máximo práctico de $\mathrm{P}_{2} \mathrm{O}_{5}$ que es admisible en el cemento portland, que si ha de ser de calidad aceptable deberá suponer alrededor de un 2,5 por ciento. Sobrepasado este límite, el tiempo de fraguado resultante es inadmisiblemente largo y las resistencias iniciales (a 1 y 3 días) muy bajas, si bien las resistencias a largo plazo (28 días) pueden ser aceptables.

Resultados obtenidos recientemente indican que una amplia variedad de materias primas fosfáticas, en las cuales se ha determinado el factor de saturación en cal de Lea y Parker $\left[\mathrm{CaO} /\left(2,8 \mathrm{SiO}_{2}+1,18 \mathrm{Al}_{2} \mathrm{O}_{3}+0,65 \mathrm{Fe}_{2} \mathrm{O}_{3}\right)\right]$, puede dicho factor servir como guía práctica para la dosificación de la alimentación del crudo, no obstante resultar inadmisible, a efectos del contenido en $\mathrm{P}_{2} \mathrm{O}_{5}$ y fluoruros, en el estudio del equilibrio de fases a alta temperatura. Este caso de aplicabilidad del factor de saturación en cal (L.S.F.) es fortuita, puesto que la composición de los elementos mineralógicos, determinada por las ecuaciones de Bogue es incorrecta.

El examen mineralógico de los clínkeres hace patente la existencia de soluciones sólidas de silicato bicálcico combinado con $\mathrm{P}_{2} \mathrm{O}_{5}, \mathrm{Al}_{2} \mathrm{O}_{3}$ y con $\mathrm{Fe}_{2} \mathrm{O}_{3}$ y la probabilidad de que estos tengan la forma general $(\mathrm{CaO})_{2}\left(\mathrm{SiO}_{2}\right)_{1-4 \mathrm{x}}\left(\mathrm{P}_{2} \mathrm{O}_{5}\right)_{\mathrm{x}}\left(\mathrm{Al}_{2} \mathrm{O}_{3}, \mathrm{Fe}_{2} \mathrm{O}_{3}\right)_{\mathrm{x}}$; es decir se está investigando la sustitución del $\mathrm{P}^{5+}(\mathrm{Al}, \mathrm{Fe})^{3+}$ por el $2 \mathrm{Si}^{4+}$.

Se ha estudiado el comportamiento térmico de los cementos fosfáticos, mediante el empleo de una técnica calorimétrica por conductividad continua.

\section{INTRODUCCION}

En el 5. ${ }^{\circ}$ Simposio Internacional sobre Química del Cemento el Sr. Gutt presentó una comunicación referente a las investigaciones sobre la fabricación de cementos a partir de materias primas fosfáticas. Entre otras cosas dijo, que los estudios sobre el equili- 
brio de fases a alta temperatura del sistema $\mathrm{CaO}-\mathrm{SiO}_{2}-\mathrm{P}_{2} \mathrm{O}_{5}$ han proporcionado una base fundamental para el conocimiento del papel de los fosfatos. También ha sido aclarada la especial importancia de los fluoruros como mineralizadores en la fabricación de los cementos fosfáticos. En 1971 Gutt y Smith presentaron unos estudios sobre el uso del fosfogipsum, un suproducto del proceso de la fabricación, por vía húmeda, del ácido fosfórico, en la fabricación combinada para la elaboración de cemento y ácido sulfúrico, haciendo una revisión de las dificultades que surgen por la presencia de fosfatos y fluoruros en el fosfogipsum. Gutt y Nurse en su informe principal para este Simposio hacen una revisión sobre los estudios de equilibrio de fases, que vienen realizándose desde el Simposio de Tokio, respecto a los sistemas que contienen fosfatos y fluoruros. El presente trabajo abarca los resultados de los estudios hechos por la Building Research Station relativos al empleo de materias primas fosfáticas, que se han podido completar desde el $5 .^{\circ}$ Simposio.

\section{APRECIACION DE LA APTITUD DE LAS MATERIAS PRIMAS PARA LA FABRICACION DE CEMENTO}

Se ha valorado la idoneidad de tres clases de materias primas fosfáticas para la fabricación de cemento.

1) Caliza y arcilla procedente de Africa Occidental para su utilización por el proceso convencional a base de caliza y arcilla y obtener el clínker de composición, además de fosfatos y fluoruros.

2) Caliza y esquisto procedentes de Uganda para su utilización en el proceso de fabricación de clínker con alto contenido en hierro a base de arcilla y caliza.

3) Fosfogipsum para su utilización en los procesos de fabricación de cemento y ácido sulfúrico.

\section{PROCESOS DE FABRICACION A BASE DE ARCILLA/CALIZA: METODOS EXPERIMENTALES}

\section{Materias primas procedentes del Africa Occidental}

Las materias primas investigadas fueron dos calizas, cuyos contenidos en $\mathrm{P}_{2} \mathrm{O}_{5}$ fueron de 1,7 y $6,7 \%$ (expresándose en este trabajo el porcentaje del peso) y una arcilla con un contenido en $\mathrm{P}_{2} \mathrm{O}_{5}$ del $0,36 \%$. En la tabla 1 se facilitan los análisis químicos completos.

Para la primera fase de las investigaciones se preparó una serie constituida por 18 clínkeres que contenían de 2,2 a $6,0 \%$ de $\mathrm{P}_{2} \mathrm{O}_{5}$, siendo su factor en cal, calculado de acuerdo con fórmula de Lea y Parker $\left[\mathrm{CaO} /\left(2,80 \mathrm{SiO}_{2}+1,18 \mathrm{Al}_{2} \mathrm{O}_{3}+0,65 \mathrm{Fe}_{2} \mathrm{O}_{3}\right)\right]$, del orden de 1,00 a 1,06; esto se ha hecho con el fin de determinar qué influencia tiene variar el contenido de $\mathrm{P}_{2} \mathrm{O}_{5}$ combinado con la cal. La fórmula de Lea y Parker no es estrictamente aplicable a los clínkeres fosfáticos, puesto que no establece ninguna deducción referente a los efectos del $\mathrm{P}_{2} \mathrm{O}_{5}$ en el equilibrio de fases, pero proporciona un procedimiento útil para comparar los clínkeres y para dosificar las materias primas. Los clínkeres se calcinaron durante 30 minutos a $1.450^{\circ} \mathrm{C}$. En la tabla 2 se muestran la lista completa de clínkeres preparados por esta vía y sus composiciones químicas, calculadas a partir de la 
dosificación de las materias primas. En la tabla 3 se presentan los resultados de los análisis químicos de cal libre (para todos los clínkeres), de $\mathrm{P}_{2} \mathrm{O}_{5}$ y fluoruros (clínkeres con hasta un $4 \%$ de $\mathrm{P}_{2} \mathrm{O}_{5}$ ) y en la tabla 4 puede verse la composición de los compuestos, calculada a partir de la proporción de la alimentación de crudos. En la figura 1 pueden verse los gráficos de cal libre en función del $\mathrm{CaO}$ total y del LF (límite de saturación en cal) para cada tipo de clínkeres. Los clínkeres también se examinaron mediante análisis por rayos $\mathrm{X}$ y microscopía para determinar su composición de fases (tabla 5).

TAB L A 1

Análisis químico de las materias primas

\begin{tabular}{|c|c|c|c|c|c|c|}
\hline \multirow[b]{2}{*}{ Material } & \multicolumn{3}{|c|}{ AFRICA OCCIDENTAL } & \multicolumn{3}{|c|}{ UGANDA } \\
\hline & $\begin{array}{c}\text { CALIZA } \\
\text { A } \\
{ }^{2} \%\end{array}$ & $\begin{array}{c}\text { CALIZA } \\
\text { B } \\
\text { wt } \%\end{array}$ & $\begin{array}{c}\text { ARCILLA } \\
\text { wt } \%\end{array}$ & $\begin{array}{l}\text { CALIZA } \\
\text { M152 } \\
\text { wt } \%\end{array}$ & $\begin{array}{c}\text { CALIZA } \\
\text { M153 } \\
\text { wt } \%\end{array}$ & $\begin{array}{c}\text { PIZARRA } \\
\text { M151 } \\
\text { wt } \%\end{array}$ \\
\hline $\mathrm{SiO}_{2}$ & 3.06 & 2.72 & 50.74 & 3.55 & 7.31 & 61.51 \\
\hline $\mathrm{CaO}$ & 52.35 & 53.14 & 7.23 & 48.85 & 46.02 & 1.98 \\
\hline $\mathrm{MgO}$ & 0.45 & 0.29 & 5.62 & 1.20 & 0.92 & 1.23 \\
\hline $\mathrm{Fe}_{3} \mathrm{O}_{4}$ & - & - & - & 2.71 & - & - \\
\hline $\mathrm{Fe}_{2} \mathrm{O}_{3}$ & 0.44 & 0.76 & 6.22 & 3.29 & 4.00 & 7.65 \\
\hline $\mathrm{Al}_{2} \mathrm{O}_{3}$ & 1.05 & 1.53 & 12.91 & 1.40 & 16.55 & 0.72 \\
\hline $\mathrm{Mn}_{2} \mathrm{O}_{3}$ & 0.01 & 0.04 & 0.23 & 0.30 & 0.31 & 0.73 \\
\hline $\mathrm{TiO}_{2}$ & 0.06 & 0.08 & 0.87 & 0.20 & 0.33 & 0.18 \\
\hline $\mathrm{P}_{2} \mathrm{O}_{5}$ & 1.69 & $6.6 \dot{9}$ & 0.36 & 2.65 & 1.85 & 0.25 \\
\hline $\mathrm{SO}_{3}$ & 0.09 & 0.22 & 0.07 & 0.45 & 1.43 & 0.04 \\
\hline $\mathrm{Na}_{2} \mathrm{O}$ & 0.06 & 0.12 & 0.16 & 0.22 & 0.59 & 1.96 \\
\hline $\mathrm{K}_{2} \mathrm{O}$ & 0.07 & 0.05 & 0.66 & 0.34 & 1.09 & 2.56 \\
\hline $\mathrm{F}$ & 0.21 & 0.83 & 0.17 & 0.23 & 0.34 & 0.08 \\
\hline $\mathrm{Cl}$ & - & - & 0.04 & - & - & - \\
\hline$s^{2-}$ & - & - & - & 0.05 & 0.12 & - \\
\hline $\mathrm{SrO}$ & 0.04 & 0.04 & - & 0.44 & 0.59 & 0.03 \\
\hline $\mathrm{BaO}$ & - & - & - & - & - & 0.11 \\
\hline $\mathrm{H}_{2} \mathrm{O}$ & $(0.69)$ & $(0.96)$ & $(8.72)$ & - & - & - \\
\hline P.F. $\left(1000^{\circ} \mathrm{C}\right)$ & 40.14 & 34.79 & 14.63 & 35.27 & 33.72 & 4.86 \\
\hline $\mathrm{O}_{2}$ equiv. poro $\mathbf{F}$ & -0.09 & -0.35 & -0.07 & -0.10 & -0.14 & - \\
\hline TOTAL & 99.63 & 100.95 & 99.84 & 100.37 & 99.88 & 99.72 \\
\hline $\mathrm{F} / \mathrm{P}_{2} \mathrm{O}_{5}$ & 0.12 & 0.12 & 0.47 & 0.09 & 0.18 & 0.32 \\
\hline
\end{tabular}

Estos resultados se refieren a material secado a $110^{\circ} \mathrm{C}$. 


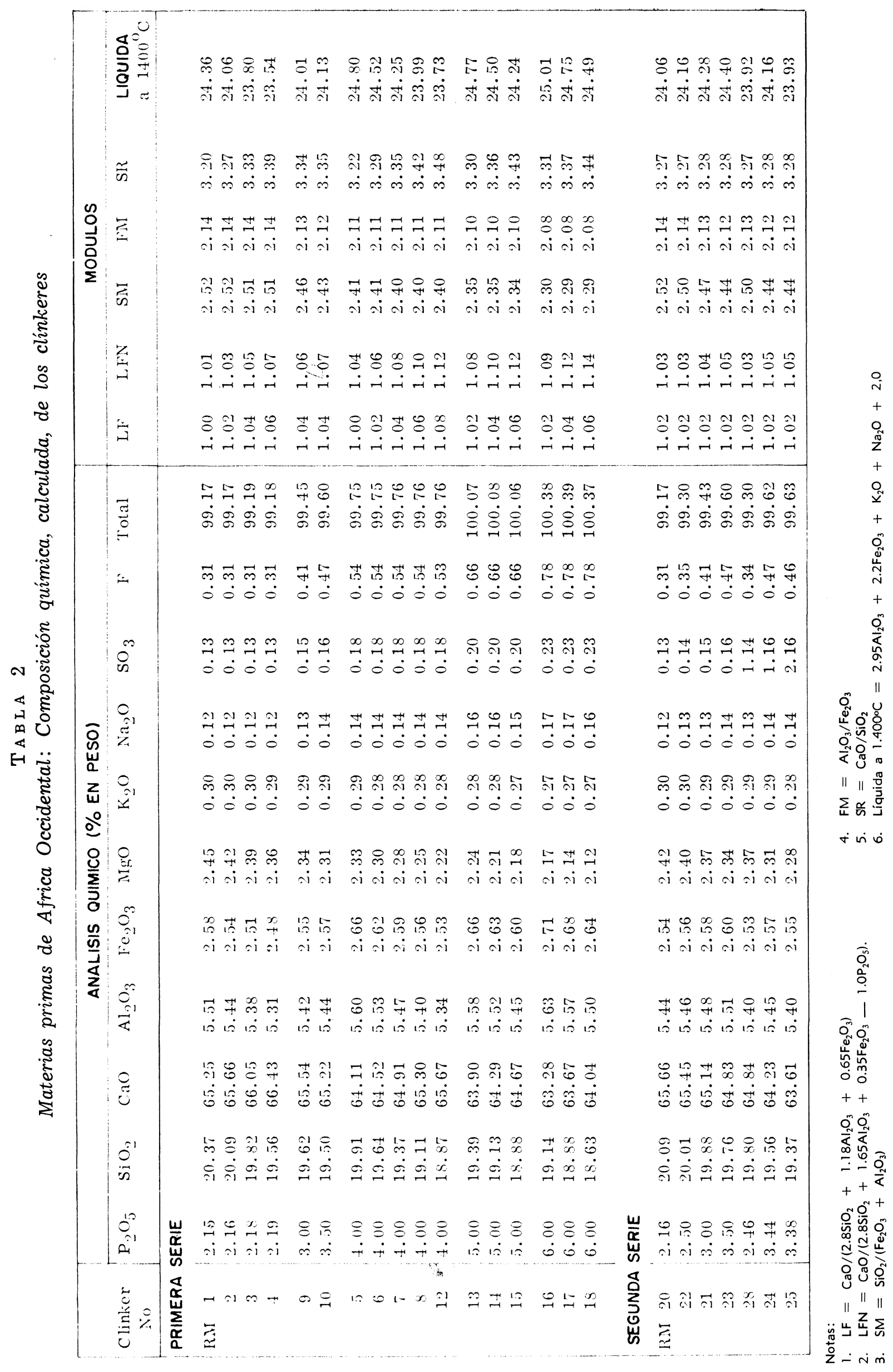


T A B L A 3

Materias primas de Africa Occidental: Resultados de los análisis químicos de los clinkeres

\begin{tabular}{|c|c|c|c|c|c|c|c|c|}
\hline \multirow{3}{*}{$\begin{array}{c}\text { Clinker } \\
\text { No }\end{array}$} & \multirow{3}{*}{$\mathrm{LF}$} & \multicolumn{5}{|c|}{ ANALISIS DEL CLINKER ( $\%$ EN PESO) } & \multicolumn{2}{|c|}{$\mathrm{F} / \mathrm{P}_{2} \mathrm{O}_{5}$} \\
\hline & & \multirow{2}{*}{$\begin{array}{l}\text { CALCULADO } \\
\mathrm{P}_{2} \mathrm{O}_{5}\end{array}$} & \multicolumn{4}{|c|}{ Experimental } & \multirow[t]{2}{*}{ CALCULADO } & \multirow[t]{2}{*}{ Experimental } \\
\hline & & & $\mathrm{P}_{2} \mathrm{O}_{5}$ & $\mathrm{~F}$ & $\mathrm{SO}_{3}$ & COO LIBRE & & \\
\hline \multicolumn{2}{|c|}{ PRIMERA SERIE } & & & & & & & \\
\hline RM 1 & 1.00 & 2.15 & 2.25 & 0.29 & $\mathrm{ND}$ & NIL & 0.144 & 0.129 \\
\hline 2 & 1.02 & 2.16 & 2.26 & 0.23 & ND & 0.04 & 0.144 & 0.102 \\
\hline 3 & 1.04 & 2.18 & 2.27 & 0.30 & ND & 0.6 & 0.142 & 0.132 \\
\hline 4 & 1.06 & 2.19 & 2.27 & 0.32 & ND & 0.8 & 0.142 & 0.140 \\
\hline 9 & 1.04 & 3.00 & 3.05 & 0.44 & ND & 0.2 & 0.137 & 0.144 \\
\hline 10 & 1.04 & 3.50 & 3.57 & 0.47 & ND & 0.3 & 0.134 & 0.132 \\
\hline 5 & 1.00 & 4.00 & 4.00 & 0.44 & ND & NIL & 0.134 & 0.110 \\
\hline 6 & 1.02 & 4.00 & 4.00 & 0.54 & ND & NIL & 0.134 & 0.135 \\
\hline 7 & 1.04 & 4.00 & 4.02 & 0.59 & ND & 0.4 & 0.134 & 0.147 \\
\hline 8 & 1.06 & 4.00 & 4.05 & 0.53 & ND & ND & 0.134 & 0.131 \\
\hline 12 & 1.08 & 4.00 & ND & ND & ND & 1.2 & - & - \\
\hline 13 & 1.02 & 5.00 & $\mathrm{ND}$ & $\mathrm{ND}$ & ND & NIL & 0.132 & - \\
\hline 14 & 1.04 & 5.00 & ND & ND & ND & 0.4 & 0.132 & - \\
\hline 15 & 1.06 & 5.00 & ND & ND & ND & 0.8 & 0.132 & - \\
\hline 16 & 1.02 & 6.00 & ND & $\mathrm{ND}$ & ND & 0.03 & 0.130 & - \\
\hline 17 & 1.04 & 6.00 & $\mathrm{ND}$ & ND & ND & 0.5 & 0.130 & - \\
\hline 18 & 1.06 & 6.00 & ND & ND & ND & ND & 0.130 & - \\
\hline \multicolumn{9}{|c|}{ SEGUNDA SERIE } \\
\hline 20 & 1.02 & 2.15 & 2.14 & 0.24 & $<0.05$ & 0.5 & 0.144 & 0.112 \\
\hline 22 & 1.02 & 2.50 & 2.49 & 0.22 & 0.06 & 0.2 & 0.142 & 0.088 \\
\hline 21 & 1.02 & 3.00 & 2.97 & 0.31 & $<0.05$ & 0.3 & 0.118 & 0.104 \\
\hline 23 & 1.02 & 3.50 & 3.38 & 0.35 & 0.12 & 0.2 & 0.101 & 0.104 \\
\hline 28 & 1.02 & 2.46 & 2.43 & 0.38 & 0.68 & 0.6 & 0.143 & 0.156 \\
\hline 24 & 1.02 & 3.44 & 3.35 & 0.35 & 0.97 & 0.2 & 0.102 & 0.105 \\
\hline 25 & 1.02 & 3.38 & 3.42 & 0.43 & 1.38 & 0.1 & 0.103 & 0.126 \\
\hline
\end{tabular}

ND - No determinado.

El objeto principal de la segunda serie de clínkeres fue producir suficiente cemento para poder realizar ensayos de resistencia a compresión, de acuerdo con la Norma Británica BS $12: 1958$. Todos los clínkeres se hicieron con un límite de saturación en cal $\mathrm{LF}=1,02$ puesto que la primera serie de ensayos mostraron que esto correspondía con el contenido óptimo en $\mathrm{CaO}$, independientemente del contenido en $\mathrm{P}_{2} \mathrm{O}_{5}$ (fig. 1). Se produjeron clínkeres con contenidos en $\mathrm{P}_{2} \mathrm{O}_{5}$ (calculados) del 2,2, 2,5, 3,0 y 3,5\%. Con objeto de evaluar los probables efectos del $\mathrm{SO}_{3}$ en los clínkeres, que en la práctica puede ser aportado por la combustión del fueloil, se prepararon también clínkeres que contenían 2,5 de $\mathrm{P}_{2} \mathrm{O}_{5}, 1,0$ de $\mathrm{SO}_{3} ; 3,5$ de $\mathrm{P}_{2} \mathrm{O}_{5}, 1,0$ de $\mathrm{SO}_{3} ; 3,5$ de $\mathrm{P}_{2} \mathrm{O}_{5}, 2,0$ de $\mathrm{SO}_{3}$ (calculados). Las composiciones químicas calculadas y las características técnicas de los clínkeres pueden verse en la tabla 2 . Los clínkeres se calcinaron a $1.450^{\circ} \mathrm{C}$ durante 30 minutos. Se analizaron. cada uno de los clínkeres determinándose la cal libre, el $\mathrm{P}_{2} \mathrm{O}_{5}$, el $\mathrm{F}$ (y también el $\mathrm{SO}_{3}$ cuando era apropiado) y se examinaron mediante análisis por rayos $\mathrm{X}$ y por microscopio. Se pre- 
pararon cementos mediante molienda de los clínkeres, añadiéndose la suficiente cantidad de yeso para obtener un contenido total en $\mathrm{SO}_{3}$ del 1,75\%, con una superficie específica de aproximadamente $280 \mathrm{~m}^{2} / \mathrm{kg}$, determinándose las resistencias a compresión sobre probetas de mortero con relación $1: 3$, hechas de acuerdo con la Norma Británica BS $12: 1958$. Los resultados de los ensayos pueden verse en las figuras 6 y 7. Se realizaron otros ensayos de acuerdo con la Norma Británica BS 12:1958, como por ejemplo tiempo de fraguado, con carácter selectivo (tabla 6). Tres de los cementos preparados se examinaron por medio de calorimetría de conducción continua, junto con un cemento hecho a partir de materias primas de Uganda.

TAB A A 4

Composición calculada de los compuestos del clínker hecho con materiales del Africa Occidental

\begin{tabular}{|c|c|c|c|c|c|c|c|c|c|c|c|c|c|}
\hline \multirow{2}{*}{\multicolumn{2}{|c|}{$\begin{array}{c}\text { Clinker } \\
\text { No }\end{array}$}} & \multirow{2}{*}{ CONTENIDO } & \multicolumn{5}{|c|}{ BOGUE-NO ADMISIBLE EL $\mathrm{P}_{2} \mathrm{O}_{5}$} & \multicolumn{6}{|c|}{ NURSE-ADMISIBLE EL $\mathrm{P}_{2} \mathrm{O}_{5}$} \\
\hline & & & $\mathrm{C}_{4} \mathrm{AF}$ & $\mathrm{C}_{3} \mathrm{~A}$ & $\mathrm{C}_{3} \mathrm{~S}$ & & \multirow{2}{*}{$\begin{array}{l}\text { CaO } \\
\text { LIBRE }\end{array}$} & $\mathrm{C}_{4} \mathrm{AF}$ & $\mathrm{C}_{3} \mathrm{~A}$ & $\mathrm{C}_{3} \mathrm{~S}$ & PSS & $\mathrm{C}_{2} \mathrm{~S}$ & \multirow{2}{*}{$\begin{array}{r}\mathrm{CaO} \\
\text {-LIBRE }\end{array}$} \\
\hline \multicolumn{3}{|c|}{ PRIMERA SERIE } & & & & & & & & & & & \\
\hline $\mathrm{RM}$ & 1 & 2.15 & 8.36 & 10.92 & 74.84 & 5.88 & 0.00 & 8.17 & 10.68 & 48.44 & 32.09 & 0.00 & 0.62 \\
\hline & 2 & 2.16 & 8.25 & 10.79 & 79.37 & 1.59 & 0.00 & 8.06 & 10.55 & 47.12 & 32.27 & 0.00 & 2.00 \\
\hline & 3 & 2.18 & 8.14 & 10.66 & 80.33 & 0.00 & 0.87 & 7.95 & 10.42 & 45.85 & 32.44 & 0.00 & 3.35 \\
\hline & 4 & 2.19 & 8.03 & 10.53 & 79.25 & 0.00 & 2.19 & 7.85 & 10.29 & 44.62 & 32.59 & 0.00 & 4.65 \\
\hline & 9 & 3.00 & 8.31 & 10.78 & 80.06 & 0.00 & 0.84 & 8.05 & 10.44 & 32.60 & 44.65 & 0.00 & 4.25 \\
\hline & 10 & 3.50 & 8.42 & 10.86 & 79.89 & 0.00 & 0.83 & 8.11 & 10.46 & 24.60 & 52.02 & 0.00 & 4.80 \\
\hline & 5 & 4.00 & 8.75 & 11.21 & 74.02 & 6.03 & 0.00 & 8.38 & 10.74 & 18.75 & 59.41 & 0.00 & 2.72 \\
\hline & 6 & 4.00 & 8.64 & 11.07 & 78.56 & 1.74 & 0.00 & 8.28 & 10.61 & 17.66 & 59.40 & 0.00 & 4.05 \\
\hline & 7 & 4.00 & 8.52 & 10.94 & 79.72 & 0.00 & 0.82 & 8.17 & 10.48 & 16.61 & 59.38 & 0.00 & 5.35 \\
\hline & 8 & 4.00 & 8.42 & 10.81 & 78.61 & 0.00 & 2.17 & 8.07 & 10.36 & 15.57 & 59.36 & 0.00 & 6.65 \\
\hline & 12 & 4.00 & 8.31 & 10.68 & 77.59 & 0.00 & 3.42 & 7.96 & 10.24 & 14.62 & 59.33 & 0.00 & 7.84 \\
\hline & 13 & 5.00 & 8.85 & 11.22 & 78.09 & 1.83 & 0.00 & 8.39 & 10.64 & 1.73 & 74.08 & 0.00 & 5.16 \\
\hline & 1.1 & 5.00 & 8.74 & 11.09 & 79.38 & 0.00 & 0.79 & 8.28 & 10.52 & 0.70 & 74.05 & 0.00 & 6.44 \\
\hline & 15 & 5.00 & 8.63 & 10.96 & 78.30 & 0.00 & 2.10 & 8.18 & 10.40 & -0.28 & 74.02 & 0.00 & 7.69 \\
\hline & 16 & 6.00 & 9.07 & 11.39 & 77.62 & 1.92 & 0.00 & 8.51 & 10.68 & -14.13 & 88.68 & 0.00 & 6.26 \\
\hline & 17 & 6.00 & 8.96 & 11.25 & 79.03 & 0.00 & 0.76 & 8.40 & 10.56 & -15.14 & 88.65 & 0.00 & 7.53 \\
\hline & 18 & 6.00 & 8.85 & 11.12 & 77.95 & 0.00 & 2.07 & 8.30 & 10.44 & -16.11 & 88.61 & 0.00 & 8.76 \\
\hline \multicolumn{14}{|c|}{ SEGUNDA SERIE } \\
\hline \multirow[t]{7}{*}{$R M$} & 20 & 2.16 & 8.25 & 10.79 & 79.37 & 1.59 & 0.00 & 8.06 & 10.55 & 47.12 & 32.27 & 0.00 & 2.00 \\
\hline & 22 & 2.50 & 8.32 & 10.84 & 79.23 & 1.61 & 0.00 & 8.10 & 10.56 & 41.70 & 37.26 & 0.00 & 2.38 \\
\hline & 21 & 3.00 & 8.42 & 10.92 & 79.00 & 1.66 & 0.00 & 8.16 & 10.58 & 33.67 & 44.66 & 0.00 & 2.94 \\
\hline & 23 & 3.50 & 8.53 & 10.99 & 78.78 & 1.70 & 0.00 & 8.22 & 10.59 & 25.65 & 52.04 & 0.00 & 3.50 \\
\hline & 28 & 2.46 & 8.31 & 10.82 & 79.50 & 1.37 & 0.00 & 8.09 & 10.54 & 41.95 & 36.98 & 0.00 & 2.44 \\
\hline & 24 & 3.44 & 8.52 & 10.97 & 79.24 & 1.28 & 0.00 & 8.21 & 10.57 & 25.99 & 51.63 & 0.00 & 3.60 \\
\hline & 25 & 3.38 & 8.52 & 10.97 & 79.08 & 1.44 & 0.00 & 8.21 & 10.57 & 26.44 & 51.26 & 0.00 & 3.52 \\
\hline
\end{tabular}

Notas: 1. Las composiciones de los compuestos se han deducido por la aplicación directa de las ecuaciones, ajustadas a $100 \%$.

2. Véase en el texto las exactas ecuaciones usadas en los cálculos. 
Materias primas del Africa Occidental. Resultados del recuento de puntos por microscopía de las secciones pulimentadas

\begin{tabular}{|c|c|ccc|}
\hline RNI & $\mathrm{P}_{2} \mathrm{O}_{\bar{j}}$ & \multicolumn{3}{|c|}{ FASES PRINCIPALES (\% EN PESO) } \\
\cline { 3 - 5 } No & $\%$, PESO & ALITA & BELITA & INTERSTICIAL \\
\hline 20 & 2.16 & 60 & 24 & 16 \\
2 & 2.16 & 63 & 23 & 14 \\
9 & 3.0 & 59 & 31 & 10 \\
10 & 3.5 & 54 & 33 & 12 \\
7 & 4.0 & 45 & 43 & 12 \\
13 & 5.0 & 32 & 59 & 9 \\
16 & 6.0 & 23 & 68 & 9 \\
\hline
\end{tabular}

T A B L A 6

Resultados de los ensayos según la BS 12:1958

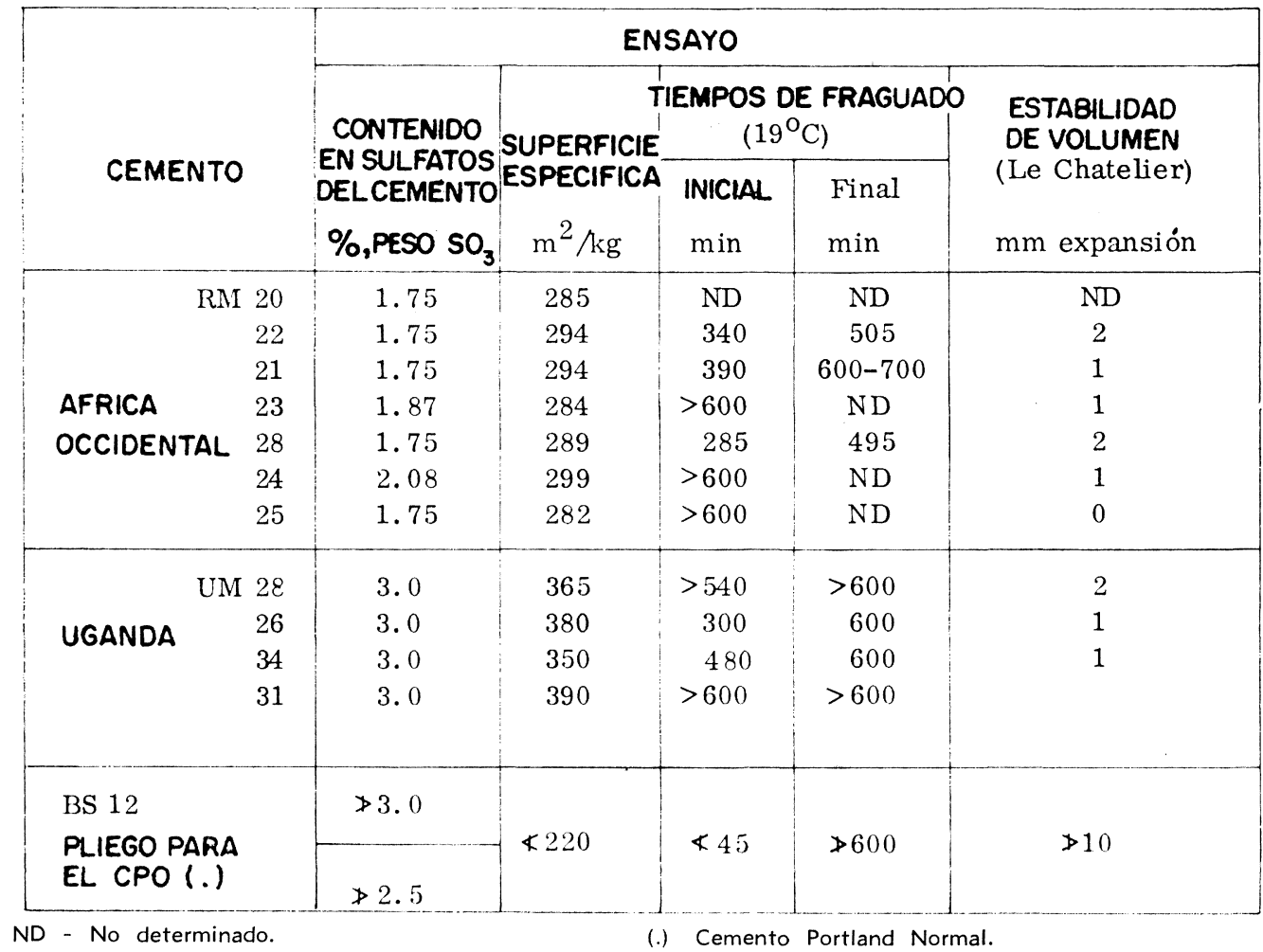

\section{Materias primas procedentes de Uganda}

La industria cementera de Uganda produce un cemento de buena calidad desde 1952 en Tororo a partir de materias primas fosfáticas. La industria se ha basado en la explotación de un depósito de caliza fosfática, que previamente se había considerado inadecuada para la fabricación de cemento, debido al efecto perjudicial del fosfato. Un reciente trabajo de Nurse (4) estableció que un 2,5\% de $\mathrm{P}_{2} \mathrm{O}_{5}$ era el nivel máximo de fosfatos que podía tolerarse en un cemento hecho con estos materiales. Este descubrimiento se ha fundamentado y confirmado por medio de los detallados estudios de equilibrio de fases realizados por Gutt (5) y (6) y ratificado por las amplias experiencias prácticas llevadas a cabo en Uganda (1). Con el fin de mitigar parcialmente el nocivo efecto del fosfato se ha añadido fluorita a la alimentación de crudos de Tororo. La experiencia práctica de la UCI (Industria Cementera de Uganda), que tiene en cuenta los adversos efectos debidos a los fluoruros en sí, ha demsotrado que el contenido óptimo de fluoruros de sus 
clínkeres es de aproximadamente $0,85 \%$ de F. De una forma similar el contenido óptimo de yeso para el cemento molido ha sido establecido por la UCI como el equivalente a un total en el contenido de $\mathrm{SO}_{3}$ de aproximadamente un $3 \%$.

Sin embargo las reservas que hasta ahora se conocen de calizas con un contenido de fosfatos suficientemente bajo son limitadas y la Building Research Station ha realizado un trabajo utilizando una caliza procedente de un origen optativo con objeto de evaluar si puede ser utilizada con el fin de continuar económicamente la producción de cemento en Tororo. Los principales problemas relacionados con el uso de este depósito de caliza residen en el elevado nivel de $\mathrm{P}_{2} \mathrm{O}_{5}$ y el inesperado elevado contenido en magnetita $\left(\mathrm{Fe}_{3} \mathrm{O}_{4}\right)$.

Las materias primas de Uganda que se han estudiado son dos calizas con contenidos en $\mathrm{P}_{2} \mathrm{O}_{5}$ de 1,85 y $2,6 \%$ respectivamente y un esquisto con un contenido en $\mathrm{P}_{2} \mathrm{O}_{5}$ del $0,25 \%$. En la tabla 1 se facilitan los análisis químicos completos. Se prepararon dos series de clínkeres: (1) para determinar el máximo de cal combinable y (2) para ensayarlo según la Norma Británica BS 12:1958.

Para la primera serie de clínkeres se utilizaron estas materias primas y calcita de la clase reactiva, obteniéndose clínkeres con contenidos de $\mathrm{P}_{2} \mathrm{O}_{5}$ del orden del 2,0 al $3,4 \%$. A cada alimentación de crudo se añadió espato flúor para que las mezclas sometidas a cocción contuviesen, aproximadamente, un 0,85 \% de F. Para la dosificación de las materias primas y por considerarlo conveniente se utilizó nuevamente la fórmula de Lea y Parker de saturación en cal. Los crudos se cocieron durante 30 minutos a $1.400^{\circ} \mathrm{C}$ analizándose el $\mathrm{CaO}$ y en algunos casos el $\mathrm{P}_{2} \mathrm{O}_{5}$ y el fluoruro (tabla 7). También se examinaron por rayos $\mathrm{X}$ y al microscopio para determinar la composición mineralógica ( $\mathrm{Ta}$ bla 9). En las tablas 8 y 9 pueden verse, respectivamente, las composiciones químicas calculadas y composiciones mineralógicas de los clínkeres. En la figura 2 puede verse el contenido en $\mathrm{CaO}$ libre de los clínkeres en relación con el contenido total en $\mathrm{CaO}$ y en $\mathrm{P}_{2} \mathrm{O}_{5}$.

T A B L A 7

Materias primas de Uganda: Resultados de los análisis químicos de los clínkeres

\begin{tabular}{|c|c|c|c|c|c|c|c|}
\hline \multirow{3}{*}{$\begin{array}{l}\text { Clinker } \\
\text { UM No }\end{array}$} & \multirow{3}{*}{$\begin{array}{l}\text { LIMITE DE } \\
\text { SATURACION } \\
\text { EN CAL } \\
\text { (LF) }\end{array}$} & \multicolumn{6}{|c|}{ ANALISIS DEL CLINKER (\% EN PESO) } \\
\hline & & \multicolumn{2}{|c|}{ CALCULADO } & \multicolumn{4}{|c|}{ Experimental } \\
\hline & & $\mathrm{P}_{2} \mathrm{O}_{5}$ & $\mathrm{~F}$ & $\mathrm{P}_{2} \mathrm{O}_{5}$ & $\mathrm{~F}$ & $\mathrm{SO}_{3}$ & $\begin{array}{l}\text { COO } \\
\text { LIBRE }\end{array}$ \\
\hline \multicolumn{8}{|c|}{ PRIMERA SERIE } \\
\hline 27 & 1.04 & 2.00 & 0.85 & - & - & - & 0.25 \\
\hline 24 & 1.02 & 2.46 & 0.85 & - & - & - & 0.00 \\
\hline 25 & 1.06 & 2.49 & 0.85 & 2.44 & 0.80 & 2.21 & 0.42 \\
\hline 33 & 1.04 & 3.00 & 0.85 & - & - & - & 0.66 \\
\hline 32 & 1.05 & 3.00 & 0.85 & - & - & - & 0.83 \\
\hline 30 & 1.04 & 3.35 & 0.85 & - & - & - & 1.15 \\
\hline 29 & 1.06 & 3.36 & 0.85 & - & - & - & 2.10 \\
\hline \multicolumn{8}{|c|}{ SEGUNDA SERIE } \\
\hline 28 & 1.04 & 2.00 & 0.85 & 2.00 & 0.80 & 1.60 & 0.27 \\
\hline 26 & 1.06 & 2.49 & 0.85 & 2.44 & 1.08 & $\begin{array}{c}1.56 \\
\left(\mathrm{~S}^{2-}=0.14\right)\end{array}$ & 0.39 \\
\hline 34 & 1.04 & 3.00 & 0.85 & 2.95 & 0.90 & $\begin{array}{c}1.23 \\
\left(\mathrm{~S}^{2-}=0.02\right)\end{array}$ & 0.73 \\
\hline 31 & 1.03 & 3.34 & 0.85 & 3.33 & 0.87 & 0.73 & 0.13 \\
\hline
\end{tabular}


TAB L A 8

Materias primas de Uganda: composición química calculada de los clínkeres

\begin{tabular}{|c|c|c|c|c|c|c|c|c|c|c|c|c|c|c|c|c|c|c|c|c|}
\hline \multirow{2}{*}{$\frac{\begin{array}{c}\text { Clinker } \\
\text { No }\end{array}}{\text { UM }}$} & \multicolumn{13}{|c|}{$\begin{array}{c}\text { ANALISIS QUIMICO } \\
\text { (BASADO EN LA ALIMENTACION DEL CRUDO SIN ADICION DE ESPATO FLUOR) }\end{array}$} & \multicolumn{7}{|c|}{ MODULOS TECNICOS } \\
\hline & $\mathrm{P}_{2} \mathrm{O}_{5}$ & $\mathrm{SiO}_{2}$ & $\mathrm{CaO}$ & $\mathrm{Al}_{2} \mathrm{O}_{3}$ & $\mathrm{Fe}_{2} \mathrm{O}_{3}$ & $\mathrm{MgO}$ & $\mathrm{K}_{2} \mathrm{O}$ & $\mathrm{Na}_{2} \mathrm{O}$ & $\mathrm{SO}_{3}$ & $F$ & $\mathrm{TiO}_{2}$ & SrO & $\mathrm{Mn}_{2} \mathrm{O}_{3}$ & Total & $\mathrm{LF}$ & LFN & $S M$ & FM & $\mathrm{SR}$ & $\begin{array}{l}\text { LIQUIDO } \\
\text { a } 1400^{\circ} \mathrm{C}\end{array}$ \\
\hline \multicolumn{21}{|c|}{ PRIMERA SERIE } \\
\hline 27 & 2.00 & 18.34 & 62.51 & 4.33 & 5.55 & 1.19 & 1.60 & 0.96 & 1.52 & 0.37 & 0.45 & 0.63 & 0.38 & 99.83 & 1.04 & 1.07 & 1.85 & 0.78 & 3.41 & 28.74 \\
\hline 24 & 2.46 & 18.03 & 60.64 & 4.11 & 6.30 & 1.38 & 1.78 & 1.04 & 1.88 & 0.46 & 0.51 & 0.79 & 0.46 & 99.82 & 1.02 & 1.06 & 1.73 & 0.65 & 3.36 & 30.17 \\
\hline 25 & 2.49 & 17.51 & 61.29 & 3.96 & 6.28 & 1.38 & 1.77 & 1.03 & 1.90 & 0.46 & 0.50 & 0.79 & 0.46 & 99.82 & 1.06 & 1.11 & 1.71 & 0.63 & 3.50 & 29.67 \\
\hline 33 & 3.00 & 17.33 & 61.06 & 4.19 & 8.02 & 1.60 & 1.28 & 0.82 & 1.09 & 0.36 & 0.52 & 0.64 & 0.35 & 100.26 & 1.04 & 1.11 & 1.41 & 0.52 & 3.52 & 33.69 \\
\hline 32 & 3.00 & 17.21 & 61.22 & 4.15 & 8.00 & 1.60 & 1.28 & 0.82 & 1.11 & 0.37 & 0.52 & 0.65 & 0.36 & 100.26 & 1.05 & 1.12 & 1.42 & 0.52 & 3.56 & 33.54 \\
\hline 30 & 3.35 & 17.05 & 61.13 & 4.30 & 9.15 & 1.74 & 0.95 & 0.68 & 0.57 & 0.30 & 0.52 & 0.55 & 0.29 & 100.57 & 1.04 & 1.12 & 1.27 & 0.47 & 3.59 & 36.18 \\
\hline 29 & 3.36 & 16.79 & 61.45 & 4.22 & 9.16 & 1.75 & 0.94 & 0.67 & 0.57 & 0.30 & 0.52 & 0.56 & 0.29 & 100.57 & 1.06 & 1.14 & 1.25 & 0.46 & 3.66 & 35.96 \\
\hline \multicolumn{21}{|c|}{ SEGUNDA SERIE } \\
\hline 28 & 2.00 & 18.34 & 62.51 & 4.33 & 5.55 & 1.19 & 1.60 & 0.96 & 1.52 & 0.37 & 0.45 & 0.63 & 0.38 & 99.83 & 1.04 & 1.07 & 1.85 & 0.78 & 3.41 & $28.7 \cdot 1$ \\
\hline 26 & 2.49 & 17.51 & 61.29 & 3.96 & 6.28 & 1.38 & 1.77 & 1.03 & 1.90 & 0.46 & 0.50 & 0.79 & 0.46 & 99.82 & 1.06 & 1.11 & 1.71 & 0.63 & 3.50 & 29.67 \\
\hline 34 & 3.00 & 17.33 & 61.06 & 4.19 & 8.02 & 1.60 & 1.28 & 0.82 & 1.09 & 0.36 & 0.52 & 0.64 & 0.35 & 100.26 & 1.04 & 1.10 & 1.42 & 0.52 & 3.52 & 33.69 \\
\hline 31 & 3.34 & 17.18 & 60.96 & 4.33 & 9.15 & 1.74 & 0.95 & 0.68 & 0.57 & 0.30 & 0.52 & 0.55 & 0.29 & 100.56 & 1.03 & 1.10 & 1.27 & 0.47 & 3.54 & 36.28 \\
\hline
\end{tabular}

T A в L A 9

Materias primas de Uganda: composiciones de las fases, calculadas y experimentales, de la $2 .^{a}$ serie de clínkeres

\begin{tabular}{|c|c|c|c|c|c|c|c|c|c|c|c|c|c|c|c|c|c|}
\hline \multirow{3}{*}{$\begin{array}{l}\text { Clinker } \\
\text { CM No }\end{array}$} & \multirow{3}{*}{ 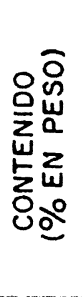 } & \multicolumn{11}{|c|}{ COMPOSICIONES CALCULADAS } & \multicolumn{5}{|c|}{ COMPOSICIONES EXPERIMENTALES } \\
\hline & & \multicolumn{5}{|c|}{$\begin{array}{l}\text { FORMULA DE BOGUE } \\
\text { SIN TOLERANCIA DE } \mathrm{P}_{2} \mathrm{O}_{5}\end{array}$} & \multicolumn{6}{|c|}{$\begin{array}{l}\text { FORMULA DE NURSE } \\
\text { CON TOLERANCIA DE } \mathrm{P}_{2} \mathrm{O}_{5}\end{array}$} & \multicolumn{4}{|c|}{$\begin{array}{l}\text { FASES IDENTIFICADAS POR CUENTA DE } \\
\text { PUNTOS AL MICROSCOPIO EN SECCION } \\
\text { PULIMENTADA (\% EN PESO) }\end{array}$} & \multirow{2}{*}{ CaO LIBRE } \\
\hline & & $\mathrm{C}_{4} \mathrm{AF}$ & $\mathrm{C}_{3} \mathrm{~A}$ & $\mathrm{C}_{3} \mathrm{~S}$ & $\mathrm{C}_{2} \mathrm{~S}$ & $\begin{array}{l}\text { COO } \\
\text { LIBRE }\end{array}$ & $\mathrm{C}_{4} \mathrm{AF}$ & $\mathrm{C}_{3} \mathrm{~A}$ & $\mathrm{C}_{3} \mathrm{~S}$ & PSS & $\mathrm{C}_{2} \mathrm{~S}$ & $\begin{array}{l}\text { CaO } \\
\text { LIBRE }\end{array}$ & ALITA & $\begin{array}{l}\mathrm{C}_{2} \mathrm{~S} \\
\text { (ATACADO } \\
\text { AZUL) }\end{array}$ & $\begin{array}{l}\text { INTERS } \\
\text { TICIAL }\end{array}$ & $\begin{array}{l}\mathrm{C}_{2} \mathrm{~S} \\
\text { (NO ATACADO) }\end{array}$ & \\
\hline 28 & 2.00 & 18.40 & 2.29 & 76.98 & 0.0 & 2.33 & 18.00 & 2.24 & 44.17 & 30.93 & 0.0 & 4.66 & 63 & 22 & 15 & - & 0.3 \\
\hline 26 & 2.44 & 21.21 & -0.17 & 74.94 & 0.0 & 4.02 & 20.63 & -0.17 & 33.69 & 38.94 & 0.0 & 6.91 & 66 & 16 & 16 & 2 & 0.4 \\
\hline 25 & 2.44 & 21.21 & -0.17 & 74.94 & 0.0 & 4.02 & 20.63 & -0.17 & 33.69 & 38.94 & 0.0 & 6.91 & 61 & 25 & 13 & 2 & - \\
\hline $3 \cdot 1$ & 2.95 & 26.64 & -2.76 & 72.96 & 0.0 & 3.16 & 25.78 & -2.66 & 24.31 & 45.98 & 0.0 & 6.59 & 38 & 38 & 22 & 2 & 0.8 \\
\hline 31 & 3.33 & 30.08 & -4.43 & 71.54 & 0.0 & 2.81 & 29.02 & -4.28 & 18.23 & 50.44 & 0.0 & 6.59 & 18 & 50 & 30 & 3 & 1.1 \\
\hline
\end{tabular}

Notas: 1. Las composiciones mineralógicas se han deducido por aplicación directa de las ecuaciones correspondientes, antes de la adición de espato fluor, y ajustadas al $100 \%$. 2. 
El principal objeto de la segunda serie de preparaciones fue producir el suficiente cemento de cada contenido de $\mathrm{P}_{2} \mathrm{O}_{5}$ para los ensayos de resistencia a compresión, tiempos de fraguado y estabilidad dimensional, realizados de acuerdo con la Norma Británica BS $12: 1958$. Se prepararon clínkeres de cemento con contenidos (calculados) de $\mathrm{P}_{2} \mathrm{O}_{5}$ del $2,0,2,5,3,0$ y $3,3 \%$ utilizando las dosificaciones de alimentación de materias primas que se consideraron ser las más adecuadas, como resultado de la primera serie de ensayos; es decir aquellas que dieron un 0,5 de $\mathrm{CaO}$ libre en el clínker. En la tabla 7 se facilitan las composiciones de la alimentación de crudos y los resultados de los análisis químicos selectivos para los clínkeres. En la tabla 8 pueden verse las composiciones químicas calculadas y los módulos técnicos, basados en los análisis de las materias primas de la alimentación sin la adición de espato flúor. $\mathrm{El}$ deseado nivel del $0,85 \%$ de $\mathrm{F}$ en los clínkeres se consiguió con la adición de $\mathrm{CaF}_{2}$ en la alimentación del crudo. Los clínkeres se examinaron por microscopía con luz reflejada y se analizaron por rayos X. La composición de las fases, calculadas mediante la aplicación de las fórmulas de Bogue (sin tolerancia para el $\mathrm{P}_{2} \mathrm{O}_{5}$ ) y por la de Nurse (con tolerancia para el $\mathrm{P}_{2} \mathrm{O}_{5}$ ) son facilitados en la tabla 9, comparándose los resultados obtenidos de la cuenta de puntos por microscopía. El clínker (UM 26) con un contenido aproximado del $2,5 \%$ de $\mathrm{P}_{2} \mathrm{O}_{5}$ fue completamente analizado, comparándose los resultados con los análisis calculados de las composiciones de las materias primas de los crudos y que pueden verse en la tabla 10.

T A B L A 10

Análisis químico, calculado $y$ experimental del clínker UM 26 hecho con materias primas de Uganda

\begin{tabular}{|c|c|c|}
\hline & CALCULADO & Experimental \\
\hline $\mathrm{SiO}_{2}$ & 17.37 & 17.83 \\
\hline $\mathrm{CaO}$ & 61.37 & 61.40 \\
\hline $\mathrm{Al}_{2} \mathrm{O}_{3}$ & 3.93 & 4.05 \\
\hline $\mathrm{Fe}_{2} \mathrm{O}_{3}$ & 6.23 & 6.52 \\
\hline $\mathrm{MgO}$ & 1.37 & 1.31 \\
\hline $\mathrm{K}_{2} \mathrm{O}$ & 1.76 & 1.36 \\
\hline $\mathrm{Na}_{2} \mathrm{O}$ & 1.02 & 0.79 \\
\hline $\mathrm{SO}_{3}$ & 1.89 & 1.56 \\
\hline $\mathrm{s}^{2-}$ & - & 0.14 \\
\hline $\mathrm{F}$ & 0.85 & 1.08 \\
\hline $\mathrm{TiO}_{2}$ & 0.50 & 0.40 \\
\hline $\mathrm{SrO}$ & 0.78 & - \\
\hline $\mathrm{Mn}_{2} \mathrm{O}_{3}$ & 0.46 & 0.46 \\
\hline $\mathrm{P}_{2} \mathrm{O}_{5}$ & 2.47 & 2.44 \\
\hline LOI * & - & 1.07 \\
\hline Pérd. de $\mathrm{O}_{2}$ equiv de $\mathrm{F}$ & -0.35 & -0.45 \\
\hline TOTAL & 99.65 & 99.96 \\
\hline
\end{tabular}

* PERDIDA AL FUEGO

Para hacer los ensayos según la BS 12:1958 se molieron los clínkeres con la suficiente cantidad de yeso para dar un $3,0 \%$ de $\mathrm{SO}_{3}$ en el cemento y con una superficie específica de $350-390 \mathrm{~m}^{2} / \mathrm{kg}$. Se observará que un $3,0 \%$ de $\mathrm{SO}_{3}$ es ligeramente superior al de la BS 12:1958 del $25 \%$ en $\mathrm{SO}_{3}$ del total de azufre para los cementos tales como estos que contienen menos de un $7 \%$ en peso de $\mathrm{C}_{3} \mathrm{~A}$. En la figura 8 se muestran los resultados de los ensayos de resistencia a compresión. En la tabla 6 pueden verse los resultados de los tiempos de fraguado y de estabilidad dimensional. La resistencia a compresión se determinó sobre cubos de 70,6 mm de mortero $1: 3$ a 1, 3, 7 y 28 días. Se experimentaron grandes dificultades al desmoldar los cubos de mortero a las 24 horas, debido a sus bajas resistencias; los cubos hechos con cemento que contenía 2,5\% de $\mathrm{P}_{2} \mathrm{O}_{5}$ se retiraron a las 48 horas (excepto 2 que fueron ensayados a las 24 horas) y los cubos que se hicieron con cementos con 2,95 y $3,33 \%$ de $\mathrm{P}_{2} \mathrm{O}_{5}$ se desmoldaron a los tres días, habiendo sido almacenados en atmósefra húmeda.

\section{TRATAMIENTO DE LA ARCILLA/CALIZA: COMPOSICION DE FASES Y COMBINACIONES}

\section{Materiales del Africa Occidental}

La primera serie de ensayos indicó que los clínkeres con un factor de saturación en cal (LF) tan alto como 1,06 podían ser aceptables, con un contenido en $\mathrm{CaO}$ libre no supe- 
rior a un $1 \%$. Los resultados de los análisis de $\mathrm{CaO}$ libre eran concordes con los clínkeres que habían sido completamente reactivos, es decir la $\mathrm{CaO}$ libre determinada no era consecuencia de la falta de homogeneidad de la alimentación del crudo.

El "punto de discontinuidad" en el gráfico de cal libre en función del límite de saturación en cal y $\mathrm{CaO}$ total (fig. 1), para clínkeres con contenidos en $\mathrm{P}_{2} \mathrm{O}_{5}$ de 2,2, 4,0, 5,0 y 6,0, siempre se presenta para un LF (límite de saturación en cal) igual a 1,02 y para los límites de saturación en cal superiores a 1,02 hay un progresivo incremento en el $\mathrm{CaO}$ no combinado. La forma en los cuatro gráficos es muy similar y se logra un contenido en $\mathrm{CaO}$ libre del $1,0 \%$ en cada uno con aproximadamente un $\mathrm{LF}=1,06$. El contenido en $\mathrm{CaO}$ de los clínkeres con factor de saturación en $\mathrm{CaO}$ constante, sin embargo, decrece cuando aumenta el contenido en $\mathrm{P}_{2} \mathrm{O}_{5}$, de modo que la cal combinable total está comprendida desde alrededor de un 65,6\%, para un contenido de $\mathrm{P}_{2} \mathrm{O}_{5}$ del $2,16 \%$ a un $63,3 \%$ para un contenido de $\mathrm{P}_{2} \mathrm{O}_{5}$ del 6,0\% (es decir aproximadamente un 0,6\% de $\mathrm{CaO}$ combinada por cada $1,0 \%$ de $\mathrm{P}_{2} \mathrm{O}_{5}$ ) (fig. 3) pero esta reducción es simplemente por dilución. La diferencia calculada en el contenido de $\mathrm{CaO}$ de los clínkeres con factores de saturación en cal (LF) de 1,02 y 1,06 ies del $0,8 \%$ (la variación en el contenido de $\mathrm{P}_{2} \mathrm{O}_{5}$ no debe alterar esta cifra) que es muy acorde con el promedio de contenido de $\mathrm{CaO}$ libre $(0,9 \%)$ de los clínkeres con un factor de saturación en cal $\mathrm{LF}=1,06$ indicado en las figuras $1 \mathrm{a}, \mathrm{b}, \mathrm{c}, \mathrm{y}$ d. El valor medio experimental es del $0,6 \%$. Así, en la práctica la fórmula de Lea y Parker puede proporcionar una fórmula de dosificación satisfactoria para obtener el mínimo de cal libre. El éxito de la fórmula de Lea y Parker, con tolerancia para el $\mathrm{P}_{2} \mathrm{O}_{5}$ o para $\mathrm{F}$, es sin embargo fortuito, puesto que el examen microscópico (tabla 5) reveló una desviación significativa con crecientes contenidos de $\mathrm{P}_{2} \mathrm{O}_{5}$ a partir de las composiciones de los compuestos, pronosticadas por las ecuaciones de Bogue, (ecuaciones 1 a 4 que se facilitan más adelante), aunque no con la amplitud pronosticada por las ecuaciones de Nurse (ecuaciones 5 a 7 facilitadas después). Las principales fases identificadas por el análisis con rayos $\mathrm{X}$ fueron alita, $\beta-C_{2} \mathrm{~S}, \alpha^{\prime}-\mathrm{C}_{2} \mathrm{~S}$ y solución sólida de ferrito aluminato cálcico (aproximadamente a $\left.\mathrm{C}_{6} \mathrm{~A}_{2} \mathrm{~F}\right)$. También es casi cierto que se encuentra presente, pero en cantidades inferiores a las detectables mediante análisis con rayos $\mathrm{X}$.

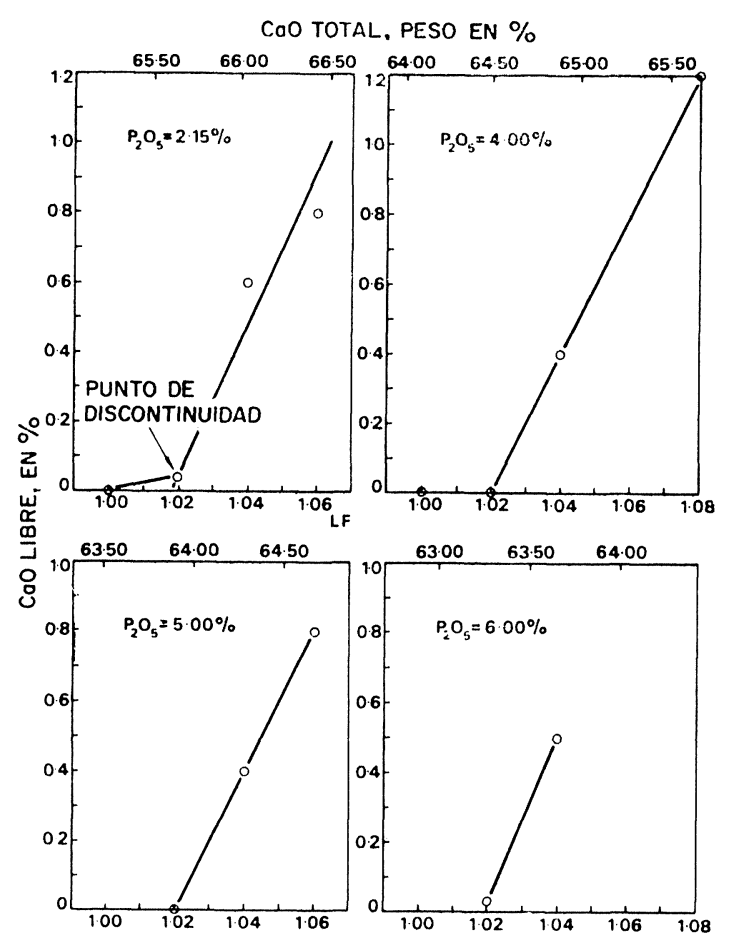

Fig. 1.-Materias primas de Africa Occidental: variación de la $\mathrm{CaO}$ libre con $\mathrm{CaO}$ total y factor en cal.

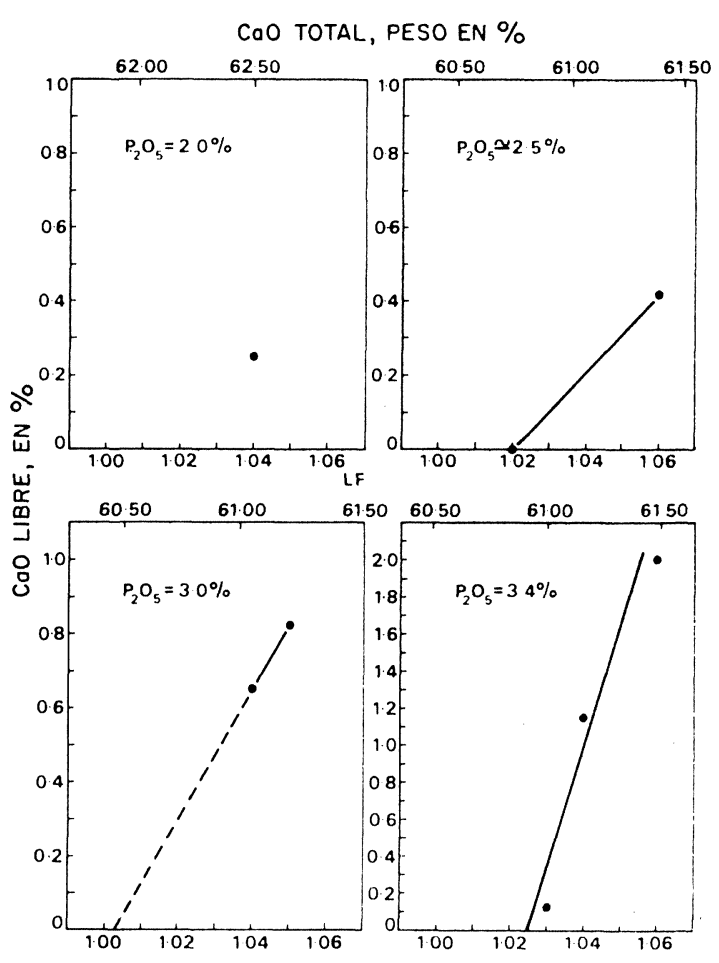

Fig. 2.-Materias primas de Uganda: Variación del $\mathrm{CaO}$ libre con $\mathrm{CaO}$ total y factor en cal. 


$$
\begin{aligned}
& \begin{array}{c|l}
\text { Ecuaciones } & \mathrm{C}_{4} \mathrm{AF}=3.04 \mathrm{Fe}_{2} \mathrm{O}_{3} \\
\text { de } & \mathrm{C}_{3} \mathrm{~A}=2.65 \mathrm{Al}_{2} \mathrm{O}_{3}-1.69 \mathrm{Fe}_{2} \mathrm{O}_{3}
\end{array} \\
& \text { Bogue } \\
& \mathrm{C}_{3} \mathrm{~S}=4.07 \mathrm{CaO}-7.60 \mathrm{SiO}_{2}-6.72 \mathrm{Al}_{2} \mathrm{O}_{3}-1.43 \mathrm{Fe}_{2} \mathrm{O}_{3} \\
& \mathrm{C}_{2} \mathrm{~S}=8.60 \mathrm{SiO}_{2}-3.07 \mathrm{CaO}+5.10 \mathrm{Al}_{2} \mathrm{O}_{3}+1.08 \mathrm{Fe}_{2} \mathrm{O}_{3} \\
& \text { Ecuaciones } \mid \mathrm{C}_{3} \mathrm{~S}=4.07 \mathrm{CaO}-7.60 \mathrm{SiO}_{2}-6.72 \mathrm{Al}_{2} \mathrm{O}_{3}-1.43 \mathrm{Fe}_{2} \mathrm{O}_{3}-9.9 \mathrm{P}_{2} \mathrm{O}_{5} \\
& \text { de } \quad \mathrm{C}_{2} \mathrm{~S}=8.60 \mathrm{SiO}_{2}-3.07 \mathrm{CaO}+5.10 \mathrm{Al}_{2} \mathrm{O}_{3}+1.08 \mathrm{Fe}_{2} \mathrm{O}_{3}-3.4 \mathrm{P}_{2} \mathrm{O}_{5} \\
& \text { Nurse } \\
& \mathrm{PSS}=14.3 \mathrm{P}_{2} \mathrm{O}_{5}
\end{aligned}
$$

Estas ecuaciones pueden aplicarse únicamente a clínkeres con límites de saturación en cal $\mathrm{LF} \leqslant 1,00$. Si el $\mathrm{LF}$ es mayor que 1,00 , entonces el $\mathrm{C}_{2} \mathrm{~S}=0,0$ y el $\mathrm{C}_{3} \mathrm{~S}=3,80 \mathrm{SiO}_{2}$ y la $\mathrm{CaO}$ libre $=\mathrm{CaO}-(\mathrm{CaO}$ combinada en otras fases $)-2,80 \mathrm{SiO}_{2}$, siendo el $\mathrm{SiO}_{2}=\mathrm{SiO}_{2}$ total si no está presente el $\mathrm{P}_{2} \mathrm{O}_{5}$ o si está presente el $\mathrm{P}_{2} \mathrm{O}_{5}\left[\mathrm{SiO}_{2}-\mathrm{SiO}_{2}\right.$ en solución sólida fosfática (PSS)]. Para los clínkeres con un LF $=1,02$ (fig. 4) la variación en la composición de los compuestos determinada experimentalmente con contenido en $\mathrm{P}_{2} \mathrm{O}_{5}$ puede expresarse por las ecuaciones siguientes:

$\begin{array}{ll}\text { Alita } & =83,292-10,128 \mathrm{P}_{2} \mathrm{O}_{5} \\ \text { Belita } & =-1,877+11,875 \mathrm{P}_{2} \mathrm{O}_{5} \\ \text { Fase intersticial } & =18,584-1,719 \mathrm{P}_{2} \mathrm{O}_{5}\end{array}$

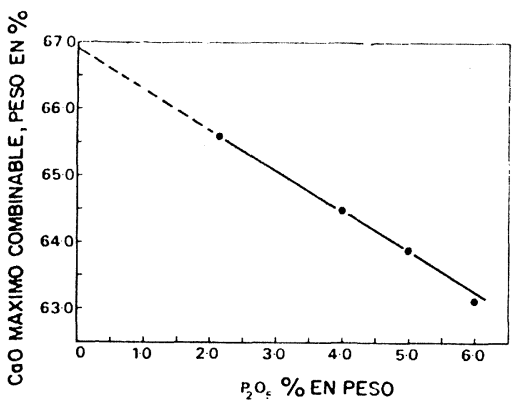

Fig. 3.-Materias primas del Africa Occidental: relación entre el máximo $\mathrm{CaO}$ combinable $y$ el contenido de $\mathrm{P}_{2} \mathrm{O}_{5}$ de los clínkeres.

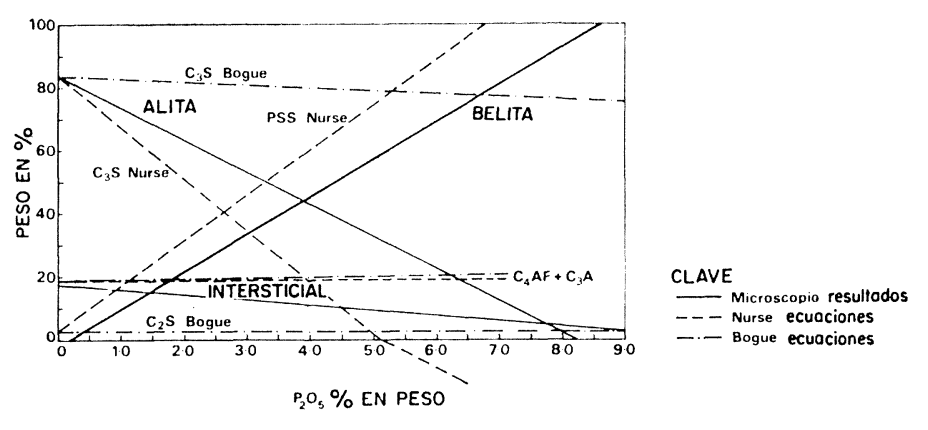

Fig. 4.-Materias primas del Africa Occiental: variación de la composición de las fases de los clinkeres con $L F=1,02$.

La detención en la parte negativa de la línea que expresa el contenido de belita indica que algo de cal libre existiría en un clínker con ausencia de $\mathrm{P}_{2} \mathrm{O}_{5}$, si fuese posible hacer una tal clase de clínker a partir de estas materias primas. La reducción en el contenido intersticial a medida que se incrementa el contenido en $\mathrm{P}_{2} \mathrm{O}_{5}$ sugiere que se llega a producir cierta combinación del $\mathrm{Al}_{2} \mathrm{O}_{3}$ o con el $\mathrm{Fe}_{2} \mathrm{O}_{3}$, o con ambos, en la fase de belita fosfática. Una mayor evidencia de esto puede deducirse de lo siguiente:

1. De los análisis de los clínkeres con $2,16-6,0 \%$ de $\mathrm{P}_{2} \mathrm{O}_{5}$ es posible, debido a la variación en el contenido de $\mathrm{P}_{2} \mathrm{O}_{5}$, llegar a una composición para el clínker con nada en el porcentaje de $\mathrm{P}_{2} \mathrm{O}_{5}$, de $20,626 \%$ de $\mathrm{SiO}_{2}$, de $66,999 \%$ de $\mathrm{CaO}, 5,333 \%$ de $\mathrm{Al}_{2} \mathrm{O}_{3}$, de $2,445 \%$ de $\mathrm{Fe}_{2} \mathrm{O}_{3}$, referido a los cuatro óxidos más importantes.

2. Si ahora se aplican las ecuaciones de Bogue normales, y los resultados de estos cálculos los ajustamos a 100, bajo el supuesto de una igual distribución de todos los componentes menores entre las fases, se obtendrá la siguiente composición de fases: 7,79\% de $\mathrm{C}_{4} \mathrm{AF}, 10,48 \%$ de $\mathrm{C}_{3} \mathrm{~A}, 80,29 \%$ de $\mathrm{C}_{3} \mathrm{~S}, 1,61 \%$ de $\mathrm{C}_{2} \mathrm{~S}$. Esta es admisiblemente próxima a la composición deducida por la cuenta de puntos en microscopía (que es de 
$81,76 \%$ de alita, 18,24 \% de intersticial) corregida para el, aparentemente, contenido negativo de belita. Es poco probable que se produzcan mayores inexactitudes si se supone que la alita es, de hecho, $\mathrm{C}_{3} \mathrm{~S}$ puro.

3. Bajo esta hipótesis la fase intersticial, para un $0,0 \%$ de $\mathrm{P}_{2} \mathrm{O}_{5}$, tendrá que tener en conjunto una composición de $30,644 \%$ de $\mathrm{Al}_{2} \mathrm{O}_{3}, 14,050 \%$ de $\mathrm{Fe}_{2} \mathrm{O}_{3}, 54,736 \%$ de $\mathrm{CaO}, 0,570 \%$ de $\mathrm{SiO}_{2}$.

4. Considerando ahora un clínker con un $8,0 \%$ de $\mathrm{P}_{2} \mathrm{O}_{5}$, aplicamos las ecuaciones [8] a [10] (que dan 2,264 \% de alita, $92,905 \%$ de belita, 4,831 de fase intersticial) y si se supone (a) que los componentes secundarios están equitativamente distribuidos entre todas las fases, (b) que la composición de la fase intersticial es constante, (c) que la alita $=\mathrm{C}_{3} \mathrm{~S}$ puro; puede demostrarse que la fase belita en este punto tiene la composición: 8,617\% de $\mathrm{P}_{2} \mathrm{O}_{5}, 20,041$ de $\mathrm{SiO}_{2}, 64,269 \%$ de $\mathrm{CaO}, 4,768$ de $\mathrm{Al}_{2} \mathrm{O}_{3}, 2,273 \%$ de $\mathrm{Fe}_{2} \mathrm{O}_{3}$, o expresado en términos molares: $\mathrm{C}_{1,146} \mathrm{~S}_{0,333} \mathrm{P}_{0,061} \mathrm{~A}_{0,047} \mathrm{~F}_{0,015}$ ó $\mathrm{C}_{2,000}$ $\mathrm{S}_{0,581} \mathrm{P}_{0,106}(\mathrm{~A}, \mathrm{~F})_{0,107}$.

Si el $\mathrm{SiO}_{2}$ es sustituido por el $\mathrm{P}_{2} \mathrm{O}_{5}, \mathrm{Al}_{2} \mathrm{O}_{3}$ y $\mathrm{Fe}_{2} \mathrm{O}_{3}$ se admite que es equivalente al $\mathrm{C}_{1,986} \mathrm{~S}$ y que la fórmula de la solución sólida puede ser expresada en términos generales como $\mathrm{C}_{2} \mathrm{~S}_{1-4 x} \mathrm{P}_{\mathrm{x}}(\mathrm{A}, \mathrm{F})_{\mathrm{x}}$, es decir hay una sustitución $1: 1$ del $\mathrm{Al}$ o del Fe por $\mathrm{P}$ en la red del $\mathrm{C}_{2} \mathrm{~S}$. La solución sólida también puede representarse como si fuese la unión hipotética de $\mathrm{C}_{2} \mathrm{~S}-\mathrm{C}_{8} \mathrm{P}(\mathrm{A}, \mathrm{F})$. Se han concebido un gran número de hipótesis hasta llegar a esta satisfactoria y simple fórmula, y, la existencia de esta solución sólida de $\mathrm{P}_{2} \mathrm{O}_{5}, \mathrm{Al}_{2} \mathrm{O}_{3}$ y $\mathrm{Fe}_{2} \mathrm{O}_{3}$, parece ser razonablemente consistente. Una investigación de los clínkeres mediante análisis con microscopio electrónico ha sido iniciada junto con un examen de las mezclas hecho con reactivos puros en la apropiada parte del sistema CaO- $\mathrm{SiO}_{2}-\mathrm{Al}_{2} \mathrm{O}_{3}-\mathrm{Fe}_{2} \mathrm{O}_{3}-\mathrm{P}_{2} \mathrm{O}_{5}$ para probar las nuevas hipótesis. Teniendo en cuenta la probada existencia de soluciones sólidas en la unión $\mathrm{C}_{2} \mathrm{~S}_{-} \mathrm{C}_{3} \mathrm{P}$ es probable que las soluciones sólidas encontradas en la práctica mostraran una sustitución más compleja del $\mathrm{P}_{2} \mathrm{O}_{5}$, $\mathrm{Al}_{2} \mathrm{O}_{3}$ y $\mathrm{Fe}_{2} \mathrm{O}_{3}$ que pueda ser representado por la fórmula $\mathrm{C}_{2} \mathrm{~S}_{1-4 \mathrm{x}} \mathrm{P}_{\mathrm{x}}(\mathrm{A}, \mathrm{F})_{\mathrm{x}}$.

Ante la falta de conocimiento de algún miembro extremo en las series $\mathrm{C}_{2} \mathrm{~S}_{1-4 \mathrm{x}} \mathrm{P}_{\mathrm{x}}(\mathrm{A}, \mathrm{F})_{\mathrm{x}}$ no es posible deducir incluso ecuaciones de ensayo optativas, de aquéllas deducidas por Nurse, para predecir la composición de las fases, basándose en las series de soluciones sólidas del $\mathrm{C}_{2} \mathrm{~S}-\mathrm{C}_{3} \mathrm{P}$. El problema se complica por la probabilidad de que la relación $\mathrm{Al}:$ Fe llegue a corresponder, no sin duda con ciertas limitaciones, con la relación $\mathrm{Al}: \mathrm{Fe}$ del clínker. Es necesario un completo estudio de las partes más importantes del sistema $\mathrm{CaO}-\mathrm{SiO}_{2}-\mathrm{Al}_{2} \mathrm{O}_{3}-\mathrm{Fe}_{2} \mathrm{O}_{3}-\mathrm{P}_{2} \mathrm{O}_{5}$ antes de que puedan ser preparadas tales ecuaciones. $\mathrm{La}$ presencia de fluoruros y otros componentes secundarios puede también influir sobre la naturaleza de la solución sólida formada.

\section{Materias primas de Uganda}

Las figuras $2 \mathrm{a}, \mathrm{b}$, c y d muestran la relación entre el $\mathrm{CaO}$ libre (no combinado) y la cantidad total de $\mathrm{CaO}$ para la primera serie de clínkeres. Los resultados analíticos del $\mathrm{CaO}$ libre concuerdan bien con los clínkeres que reaccionan completamente, es decir la cal libre registrada no surge por la falta de homogeneidad del crudo de alimentación. Como en las materias primas del Africa Occidental, la combinación total de la cal corresponde con factores de cal 1,00 a 1,02, observándose que, para fines prácticos, la fórmula normal de Lea y Parker puede ser utilizada para dosificar estas materias primas fosfáticas tan particulares. 
Las principales fases identificadas con análisis por rayos $\mathrm{X}$ fueron, alita $\alpha^{\prime}-\mathrm{C}_{2} \mathrm{~S}$ y solución sólida de $\mathrm{C}_{4} \mathrm{AF}$. $\mathrm{El}$ espectro de difracción por rayos $\mathrm{X}$ de la alita fue muy similar al del $\mathrm{C}_{3} \mathrm{~S}$ trigonal polimorfo, un hallazgo típico de los clínkeres de cemento hechos a partir de alimentación de crudos fosfáticos con fluoruros de Uganda. Se detectaron dos formas del $\alpha^{\prime}-\mathrm{C}_{2} \mathrm{~S}$, (a) con un espectro similar al espectro $\alpha^{\prime} \mathrm{m}$ que da el $\alpha^{\prime}-\mathrm{C}_{2} \mathrm{~S}$ estabilizado con potasio, (b) con un espectro similar al del $\alpha_{\mathrm{L}}^{\prime}$ estabilizado con fosfato ${ }^{7,8}$. La primera forma, probablemente se encontraba presente sólo en pequeñas cantidades y las intensidades del espectro de difracción por rayos $\mathrm{X}$ fueron débiles comparadas con los espectros moderadamente fuertes del $\alpha^{\prime}-\mathrm{C}_{2} \mathrm{~S}$ estabilizado con $\mathrm{P}_{2} \mathrm{O}_{5}$. Existe una correlación entre los datos de difracción por rayos $\mathrm{X}$ y los resultados cuantitativos de la cuenta de puntos que se presenta en la tabla 9. Es posible que la forma irregular de la fase de $\mathrm{C}_{2} \mathrm{~S}$, que estaba teñida en azul con vapores de $\mathrm{HF}$ y la fase redondeada, que no se tiñó, representen respectivamente, el $\alpha^{\prime} \mathrm{C}_{2} \mathrm{~S}$ estabilizado con fosfato y el $\alpha^{\prime}-\mathrm{C}_{2} \mathrm{~S}$ estabilizado con potasio, si bien este último puede ser $\mathrm{C}_{12} \mathrm{~A}_{7}$ o una fase similar.

El examen microscópico mostró un progresivo descenso en el contenido de $\mathrm{C}_{3} \mathrm{~S}$ y el correspondiente aumento en $\mathrm{C}_{2} \mathrm{~S}$ a medida que el nivel de $\mathrm{P}_{2} \mathrm{O}_{5}$ se elevaba de 2,0 a $3,3 \%$. Sin embargo, en contraste con este hallazgo en los clínkeres de Africa Occidental, el contenido intersticial aumentó acusadamente cuando aumentó el contenido en $\mathrm{P}_{2} \mathrm{O}_{5}$ (debido a la asociación del $\mathrm{Fe}_{2} \mathrm{O}_{3}$ y $\mathrm{P}_{2} \mathrm{O}_{5}$ en las calizas con alto $\mathrm{P}_{2} \mathrm{O}_{5}$ ) y el grado de solución de los silicatos en la fase intersticial (fig. 5). Los resultados de las observaciones al microscopio pueden ser representados por las ecuaciones 11 a 13.

$$
\begin{array}{ll}
\text { Alita } & =146,335-36,946 \mathrm{P}_{2} \mathrm{O}_{5} \\
\text { Belita } & =-33,784+24,984 \mathrm{P}_{2} \mathrm{O}_{5} \\
\text { Intersticial } & =-12,551+11,962 \mathrm{P}_{2} \mathrm{O}_{5}
\end{array}
$$

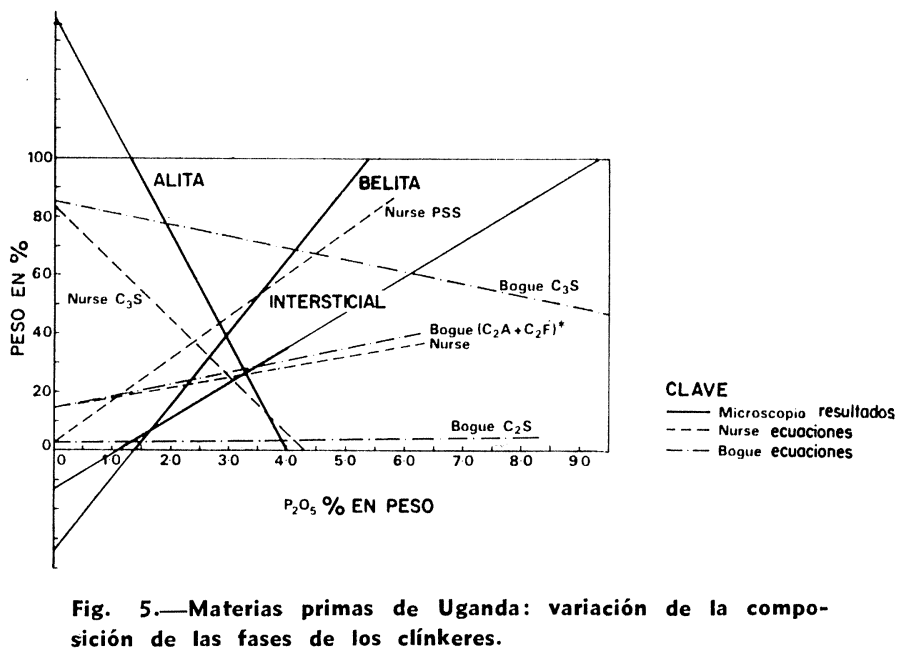

A diferencia con las ecuaciones deducidas para los clínkeres del Africa Occidental, estas ecuaciones pueden aplicarse únicamente a aquellos clínkeres examinados que tienen un determinado valor en el contenido de $\mathrm{P}_{2} \mathrm{O}_{5}$. Si se elaborasen clínkeres con menos de un $2 \%$ de $\mathrm{P}_{2} \mathrm{O}_{5}$ es probable que los contenidos de alita serían francamente próximos a los pronosticados por medio de las ecuaciones de Bogue, es decir serían más elevados que los pronosticados por las ecuaciones de Nurse. Tal comportamiento viene a confirmar la conclusión obtenida por Welch y Gutt (5) de que la adición deliberada del fluoruro cálcico a los clínkeres que contienen $2,35 \%$ de $\mathrm{P}_{2} \mathrm{O}_{5}$ tiene como resultado el que se incremente el contenido de alita. Este hallazgo ha sido confirmado en posteriores trabajos sólo en 
clínkeres de similar composición, pero los clínkeres con elevado contenido en $\mathrm{P}_{2} \mathrm{O}_{5}$ (con algo más de un $3 \%$ ) no han sido examinados, de modo que no es posible afirmar si este primer aumento en $\mathrm{C}_{3} \mathrm{~S}$ es normalmente seguido por un rápido descenso en el contenido en $\mathrm{C}_{3} \mathrm{~S}$ encontrado en los clínkeres de Uganda. El marcado incremento en $\mathrm{Fe}_{2} \mathrm{O}_{3}$ con el $\mathrm{P}_{2} \mathrm{O}_{5}$ también debe ser tenido en cuenta, puesto que se habrá incrementado la formación de líquido a temperaturas de clínkerización a medida que aumenta el $\mathrm{P}_{2} \mathrm{O}_{5}$, dando lugar a la disolución del $\mathrm{SiO}_{2}$, y probablemente también del $\mathrm{P}_{2} \mathrm{O}_{5}$ y su mantenimiento en una fase vítrea, si hay un rápido enfriamiento. Las relaciones entre las fases dominantes en los clínkeres son franca y extremadamente complejas y el sistema $\mathrm{CaO}-\mathrm{SiO}_{2}-\mathrm{P}_{2} \mathrm{O}_{5}$ $-\mathrm{Al}_{2} \mathrm{O}_{3}-\mathrm{Fe}_{2} \mathrm{O}_{3}-\mathrm{CaF}_{2}$ precisa una completa y cuidadosa investigación antes de establecerse unas reglas y pautas generales.

El análisis con sonda electrónica del clínker UM28, con un contenido total del $0,85 \%$ de $\mathrm{F}$, da el siguiente análisis aproximado para el fluoruro: $\mathrm{C}_{3} \mathrm{~S}=0,5, \mathrm{C}_{2} \mathrm{~S}=0,2$, matriz $0,2 \%$.

\section{PROCESO DE ELABORACION ARCILLA/CALIZA: RESULTADOS DE LOS ENSAYOS DE RESISTENCIA A COMPRESION}

\section{Materias primas de Africa Occidental}

El examen microscópico de la segunda serie de clínkeres hechos con materias primas del Africa Occidental muestra el mismo descenso en el contenido de $\mathrm{C}_{3} \mathrm{~S}$ con el $\mathrm{P}_{2} \mathrm{O}_{5}$ que se había observado en los clínkeres de la primera serie. Los clínkeres estaban todos bien calcinados y concordaban bastante bien los resultados de los contenidos en $\mathrm{P}_{2} \mathrm{O}_{3}$ calculados y determinados experimentalmente. En la tabla 3 se facilitan los resultados de los análisis químicos del $\mathrm{P}_{2} \mathrm{O}_{5}, \mathrm{~F}, \mathrm{SO}_{3}$ y $\mathrm{CaO}$ libre; en la tabla 6 aparecen los valores de la superficie específica, tiempos de fraguado y estabilidad dimensional, según la BS 12:1958. En la figuras 6 y 7 pueden verse los resultados de los ensayos de resistencia a la compresión.

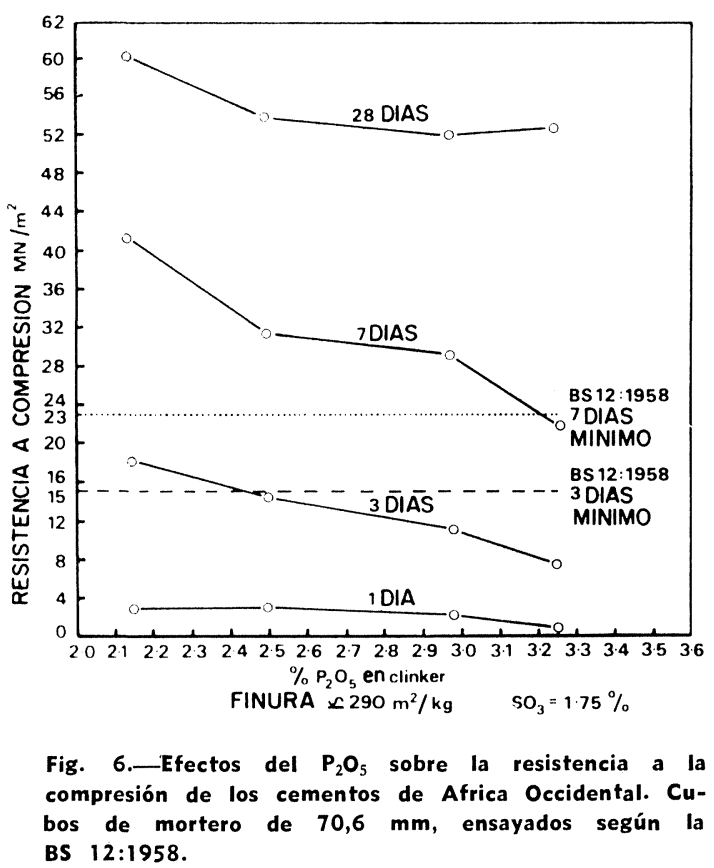

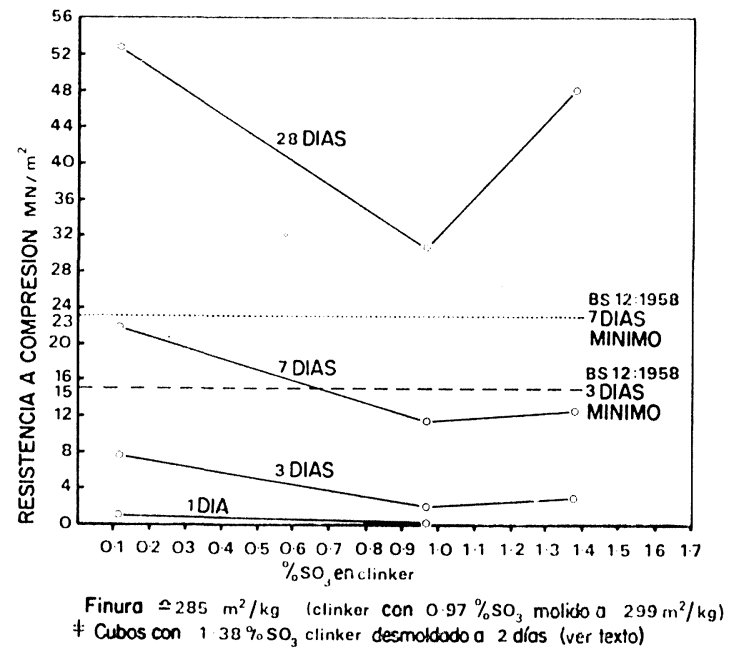

Fig. 7.-Efecto del $\mathrm{SO}_{3}$ sobre la resistencia a compresión de los cementos de Africa Occidental que contienen $3,4 \%$ de $P_{2} O_{5}$. Cubos de mortero de cemento de 70,6 mm ensayados según la BS 12:1958. 
Todos los cementos, excepto uno, dieron resistencias a 28 días comparables a las de los cementos portland normales británicos pero únicamente aquellos que tenían un contenido en $\mathrm{P}_{2} \mathrm{O}_{5}$ del 2,5 \% o incluso menos, dieron resultados totalmente satisfactorios al ser ensayados a otras edades. Aunque los cementos que contenían un 2,5\% de $\mathrm{P}_{2} \mathrm{O}_{5}$ no llegaron a cumplir las resistencias mínimas a 3 días de la Norma Británica BS 12:1958 (15 $\left.\mathrm{MN} / \mathrm{m}^{2}\right)$, sin embargo éstas podían haber alcanzado un nivel más satisfactorio si se hubiese alcanzado un grado de finura más alto, de aproximadamente $340 \mathrm{~m}^{2} / \mathrm{kg}$, que es la más frecuente en los cementos portland normales británicos. Análogamente, las resistencias de los cementos con un $3 \%$ de $\mathrm{P}_{2} \mathrm{O}_{5}$ podían mejorarse mediante una molienda más fina. Todos los cementos que contenían más de un 2,5\% de $\mathrm{P}_{2} \mathrm{O}_{5}$ no alcanzaron las resistencias mínimas y los que tenían un contenido del 3,4\% de $\mathrm{P}_{2} \mathrm{O}_{5}$ tampoco cumplieron las resistencias a 7 días.

La presencia de $\mathrm{SO}_{3}$, deliberadamente adicionado al clínker, produjo un efecto francamente nocivo sobre las resistencias a compresión de los cementos, especialmente en los ensayos a corto plazo. La presencia de un $0,7 \%$ de $\mathrm{SO}_{3}$ en el clínker que tiene un contenido del 2,5\% de $\mathrm{P}_{2} \mathrm{O}_{5}$ produjo un descenso en la resistencia a los 3 días de aproximadamente un $40 \%$. Estos resultados parecen concordar con la ya sabida sensibilidad al $\mathrm{SO}_{3}$ de los clínkeres fosfáticos fluorados (1) (5).

Todos los cementos dieron a las 24 horas resistencias extremadamente bajas (en todos los casos inferiores a $3 \mathrm{MN} / \mathrm{m}^{2}$ ), que son características en los cementos fosfáticos fluorados. Estas bajas resistencias a las 24 horas estuvieron acompañadas con tiempos de fraguado inicial no inferiores a 5,5 horas, y en el caso de cementos con más de un $3,0 \%$ de $\mathrm{P}_{2} \mathrm{O}_{5}$ el fraguado final no llegó a alcanzarse antes del límite de 10 horas. El fin del fraguado de los cementos con un $3,0 \%$ o menos de un $3 \%$ de $\mathrm{P}_{2} \mathrm{O}_{5}$ fue, aproximadamente, a las 10 horas. No se han realizado ensayos para determinar la cantidad de yeso que debe ser añadida para obtener los resultados óptimos de resistencia y tiempos de fraguado. Los efectos nocivos por la adición de " $\mathrm{SO}_{3}$ " (fig. 7) a algunos clínkeres no nos indica, necesariamente, que el hecho de incrementarse la cantidad de yeso añadido durante la molienda conducirá a un descenso en las resistencias. El efecto de los iones sulfato combinados en el clínker y procedentes de las materias primas o del combustible no siempre es comparable al efecto del sulfato incorporado durante la molienda del clínker (9).

La estabilidad dimensional, ensayada con las agujas de Le Chatelier, fue satisfactoria, sin embargo, el cemento con un alto contenido en $\mathrm{P}_{2} \mathrm{O}_{5}$ presentó una expansión notablemente alta durante el curado inicial a $19^{\circ} \mathrm{C}$.

\section{Materias primas de Uganda}

La influencia del fosfato sobre la resistencia a compresión de los cementos con un contenido constante del $3,0 \%$ de $\mathrm{SO}_{3}$ puede verse en la figura 8, que muestra la reducción de resistencias originada por el aumento en el contenido de $\mathrm{P}_{2} \mathrm{O}_{5}$, existiendo una relación casi lineal en la mayor parte de las edades del ensayo. Los cementos que contenían 2,00 y $2,44 \%$ de $\mathrm{P}_{2} \mathrm{O}_{5}$ cumplían las resistencias exigidas por la Norma Británica BS 12, mientras que el cemento con $2,9 \%$ de $\mathrm{P}_{2} \mathrm{O}_{5}$ no cumplía, por muy poco, los mínimos a 3 días, sobrepasándolos, en cambio, a los 7 días. El cemento con un $3,3 \%$ de $\mathrm{P}_{2} \mathrm{O}_{5}$ no alcanzó los valores mínimos por un amplio margen, tanto a los 3 como a los 7 días, si bien se llegó a producir una sustancial ganancia en las resistencias a edades más altas. Los dos aspectos más sobresalientes, característicos de los cementos fosfáticos fluorados, fueron sus extremadamente bajas resistencias a un día y la completa recuperación a la 
edad de alcanzar las resistencias normales, que tiene lugar al cabo de 28 días. Un cemento (con $\mathrm{P}_{2} \mathrm{O}_{5}=3,33 \%$ ) da a los 28 días resultados casi comparables a las de los cementos portland normales típicos británicos. Esta reducción de resistencias con el incremento en el $\mathrm{P}_{2} \mathrm{O}_{5}$ concuerda con los cambios en la composición de las fases (tabla 9, figura 5). A medida que el contenido en $\mathrm{C}_{3} \mathrm{~S}$ de los clínkeres decrece desde más de un $60 \%$ a menos de un $20 \%$, con el correspondiente aumento del débilmente hidráulico $\alpha^{\prime} \mathrm{C}_{2} \mathrm{~S}$ y la fase intersticial, la resistencia a 28 días, por ejemplo, cae desde $\simeq 20 \mathrm{MN} / \mathrm{m}^{2}$ a 6 $\mathrm{MN} / \mathrm{m}^{2}$.

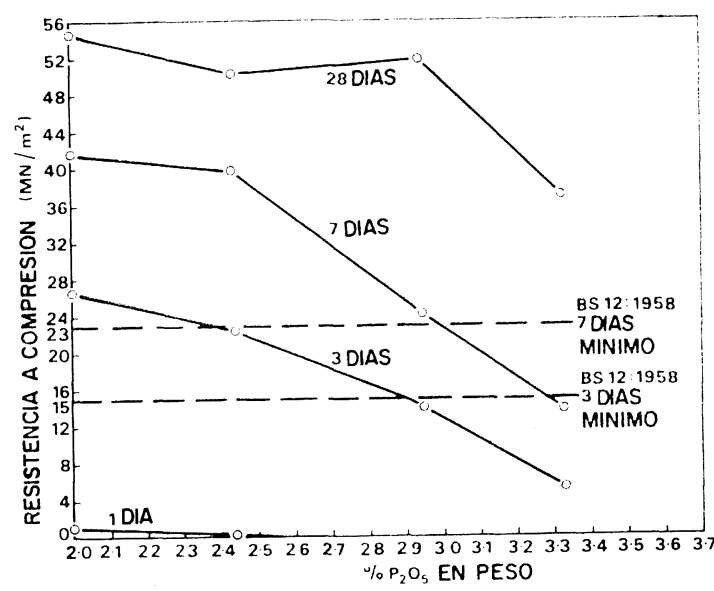

Fig. 8.-Efecto del $\mathrm{P}_{2} \mathrm{O}_{5}$ sobre la resistencia a compresión de los cementos hechos con materias primas de Uganda. Cubos de morteo de $70,6 \mathrm{~mm}$, ensayados según la BS 12:1958.

El único cemento que cumplió la exigencia de un tiempo de fin de fraguado inferior a 10 horas fue el que contenía un $2,44 \%$ de $\mathrm{P}_{2} \mathrm{O}_{5}$. Los cementos con 2,00 y $3,33 \%$ de $\mathrm{P}_{2} \mathrm{O}_{5}$ fallaron, teniendo el fraguado inicial entre las 9 y 10 horas. Esto inevitablemente justifica los problemas encontrados al desmoldar las probetas cúbicas de mortero destinadas a los ensayos de determinación de resistencias. Sin embargo, a excepción del cemento con un $2,0 \%$ de $\mathrm{P}_{2} \mathrm{O}_{5}$, los tiempos de fraguado eran mayores a medida que aumentaba el contenido en $\mathrm{P}_{2} \mathrm{O}_{5}$, como pudo comprobarse experimentalmente en los ensayos realizados por la Industria Cementera de Uganda, (UCI). Los ensayos de tiempo de fraguado se efectuaron a $18^{\circ} \mathrm{C}$ y a temperaturas de curado más altas (p. e. $30^{\circ} \mathrm{C}$ ), hecho admisible puesto que el cemento se usa bajo condiciones tropicales como las de Uganda, produciéndose el fraguado en períodos de tiempo más reducidos. Los ensayos de expansión de los tres cementos que contenían 2,0,2,5 y $3,0 \%$ de $\mathrm{P}_{2} \mathrm{O}_{5}$ y determinados con las agujas de Le Chatelier fueron satisfactorios; sin embargo, los cementos con contenidos de $\mathrm{P}_{2} \mathrm{O}_{5}$ más altos se expansionaron varios milímetros durante el curado inicial en agua a $19^{\circ} \mathrm{C}$. El cemento con $3,3 \%$ de $\mathrm{P}_{2} \mathrm{O}_{5}$ se desintegró completamente al hervir las probetas en agua, debido a su lentísima velocidad de fraguado.

Estos resultados también concuerdan y confirman los resultados obtenidos al ensayar diversas partidas de la producción de los cementos de Tororo, que se han venido realizando durante estos últimos años y que han permitido llegar a la conclusión de que los cementos que contenían hasta un 2,9\% de $\mathrm{P}_{2} \mathrm{O}_{5}$ cumplían las exigencias de las Normas Británicas.

\section{PROCESO DE FABRICACION CONJUNTA DE CEMENTO Y ACIDO SULFURICO}

El trabajo de la Building Research Station sobre la utilización del sub-producto sulfato cálcico de la fabricación del ácido fosfórico ya se describió con todo detalle precedentemente (2). Esta publicación analiza los primeros trabajos sobre el proceso de fabricación combinada de cemento y ácido sulfúrico y los problemas que surgen por la utilización del fosfogipsum en el proceso, debidos a la presencia de fosfatos y fluoruros, que fueron identificados. Se facilitó un informe de los óptimos resultados de laboratorio y de los experimentos realizados en laboratorio y en planta piloto, donde se prepararon clínkeres que contenían hasta un 1,5\% de $\mathrm{P}_{2} \mathrm{O}_{5}$. Los resultados concuerdan, generalmente, 
con los conocidos efectos del $\mathrm{P}_{2} \mathrm{O}_{5}$ y f́luoruros sobre la calidad del cemento. Los cementos obtenidos presentaban buena resistencia a largo plazo, pero con tendencia a dar bajas resistencia a 1 y 3 días. La inevitable presencia de pequeñas cantidades de sulfato residual en los clínkeres tiende a reforzar los adversos efectos de los fosfatos y fluoruros, obteniéndose por regla general cementos que no llegan a dar resistencias aprovechables, incluso a los 28 días.

Se ha llegado a la conclusión de que es posible hacer cemento portland a partir de fosfogipsum y arcilla, mediante el proceso de fabricación conjunta de cemento/ácido sulfúrico. Los requisitos esenciales son: que el fosfato debe mantenerse por debajo de un $2,5 \%$ de $\mathrm{P}_{2} \mathrm{O}_{5}$ y el flúor ha de encontrarse a un nivel óptimo, que ha de ser establecido para cada combinación de materias primas. Puesto que el sulfato residual presente en el clínker influye sobre su calidad cementicia, dicho sulfato debe estar perfectamente controlado, como realmente sucede cuando la materia prima es anhidrita. Debe también determinarse a qué nivel ha llegado la cantidad de magnesia. Probablemente sea necesaria una pequeña cantidad, que como máximo puede llegar hasta un $2 \%$. De no ser así puede obstaculizarse la formación de silicato tricálcico, por la acción combinada del aluminio y del sulfato (10). Es ciertamente evidente que es preferible la utilización del fosfogipsum, procedente de los nuevos sistemas de fabricación del ácido fosfórico (mediante los cuales se obtiene hemihidrato, o yeso con bajo contenido en fosfatos), pero pueden utilizarse los diversos tipos de fosfogipsum, resultantes de los más antiguos métodos de fabricación que contengan hasta un $1,3 \%$ de $\mathrm{P}_{2} \mathrm{O}_{5}$. Si se desean obtener los mejores resultados, el factor de saturación en cal debe ser tan alto como sea prácticamente posible. Cabe esperar resistencias relativamente bajas en el plazo de un solo día. El fluoruro presente en el fosfogipsum actuará como mineralizante de forma beneficiosa, mientras no se encuentre en excesiva cantidad y, en determinados casos, cuando el nivel de fluoruros es bajo y el nivel de fosfatos alto, la adición del $\mathrm{CaF}_{2}$ puede resultar beneficiosa.

\section{ESTUDIOS SOBRE EL COMPORTAMIENTO CALORIMETRICO DE ESTOS CEMENTOS}

Se ha estudiado el proceso evolutivo, en función del tiempo, del calor desprendido durante la hidratación de estos diversos cementos utilizando para ello un calorímetro del mismo tipo que el perfeccionado por Forrester (11) y que ya ha sido descrito por éste (9).

Con materias primas procedentes de Africa Occidental se prepararon tres cementos (RM 22,23 y 24 ) y otro cemento con materiales de Uganda (UM 11) y se procedió a su hidratación en el interior de un "calorímetro de conducción" (la temperatura a la que se realizó la hidratación fue de $25^{\circ} \mathrm{C}$ y la relación agua/cemento $=0,6$ ) y la evolución del calor desprendido durante la hidratación se estuvo anotando en el transcurso de un período de tiempo que abarcó 3 días. En la figura 9 pueden verse los resultados, observándose que llegaron a producirse considerables diferencias. En la tabla que va incluida en la figura 9 pueden verse los contenidos en fosfatos y en $\mathrm{SO}_{3}$ del clínker, así como el $\mathrm{SO}_{3}$ adicionado (en forma de yeso) de los cuatro cementos, junto con los resultados de los ensayos a compresión.

Los dos cementos, cuyos clínkeres tienen un contenido en $\mathrm{SO}_{3}$ de aproximadamente un $1 \%$ (RM 24 y UM 11) no muestran el agudo pico que aparece en los dos cementos que tienen un clínker con muy bajo $\mathrm{SO}_{3}$, aunque hay indicios de que este pico empieza a observarse en el RM 24 antes de producirse una parada en el desprendimiento de calor. No está claro si este pico es debido a una aceleración en el proceso de hidratación de la alita o de la belita, debida a la adición de yeso o a la interacción del yeso con las fases intersticiales, si bien el examen microscópico de los clínkeres hace pensar que los conte- 
nidos de $\mathrm{C}_{3} \mathrm{~A}$ fueron bajos. En los cementos RM 24 y UM 11 todo el proceso de hidratación viene fuertemente retardado y alterado si se compara con el de los cementos portland normales.
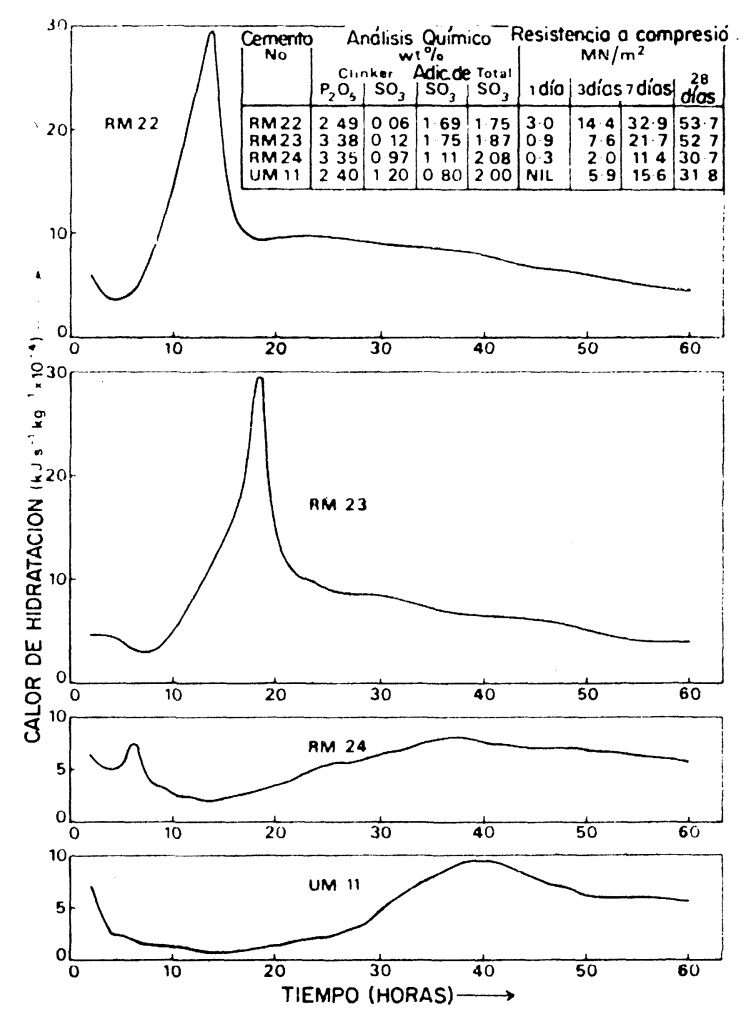

Fig. 9.-Calor de hidratación, en función del tiempo, de los cementos fosfáticos.
La influencia del fosfato puede ser apreciada comparando el cemento RM 22 (que contiene un $2,49 \%$ de $\mathrm{P}_{2} \mathrm{O}_{5}$ ) con el RM 23 (que tiene un $3,38 \%$ de $\mathrm{P}_{2} \mathrm{O}_{5}$ ). $\mathrm{El}$ incremento en el contenido de fosfatos se traduce en un retraso en la aparición del pico que se observa en los valores del calor de hidratación.

Los cuatro cementos sin excepción dieron bajas resistencias a compresión a corto plazo, siendo esto más patente en los cementos RM 24 y UM 11, que no presentaron fuertes picos de hidratación. Los cementos RM 22 y RM 24 se recuperaron dando buenas resis. tencias a los 28 días mientras que los cementos RM 24 y UM 11 no alcanzaron en el mismo grado las resistencias necesarias.

Debe recordarse, sin embargo, que el cemento UM 11, que se preparó a partir de materias primas totalmente distintas a las de los otros tres, tiene características físicas y químicas muy diferentes, señalándose también que las resistencias se obtuvieron a partir de los resultados de los ensayos sobre cubos de $12,7 \mathrm{~mm}$ hechos con mortero $1: 3$.

\section{DISCUSION Y CONCLUSIONES}

Los estudios de fabricación de cementos portland fosfáticos emprendidos a raiz del $5 .^{\circ}$ Simposio Internacional han confirmado plenamente las primitivas conclusiones (1), (2), (4), en las que se afirmaba que la máxima cantidad de $\mathrm{P}_{2} \mathrm{O}_{5}$ que puede ser tolerada en el cemento, a condición de obtener una adecuada resistencia a compresión, es, aproximadamente, del $2,5 \%$. También ha servido para confirmar la influencia que tiene la acción combinada del fosfato, fluoruro y sulfato en el clínker sobre la calidad del cemento. Mientras que los nuevos resultados confirman que el equilibrio de fases en los cementos fosfáticos puede ser explicado en muchos casos por analogía con el sistema $\mathrm{CaO}-\mathrm{SiO}_{2}$ $\mathrm{P}_{2} \mathrm{O}_{5}$ y el $\mathrm{CaO}-\mathrm{SiO}_{2}-\mathrm{P}_{2} \mathrm{O}_{5}-\mathrm{CaF}_{2}$ también se ha llegado a pensar en la posibilidad de que en algunos clínkeres puedan formarse soluciones sólidas de silicato bicálcico que contengan $\mathrm{P}_{2} \mathrm{O}_{5}$ y $\mathrm{Fe}_{2} \mathrm{O}_{3}$ combinados. Se ha iniciado una investigación sobre el subsistema $\mathrm{C}_{2} \mathrm{~S}$ en el sistema puro y, en particular, para estudiar la hipótesis de que éstos son de la forma generalizada $\mathrm{C}_{2} \mathrm{~S}_{1-4 \mathrm{x}} \mathrm{P}_{\mathrm{x}}(\mathrm{A}, \mathrm{F})_{\mathrm{x}}-\mathrm{C}_{3} \mathrm{P}$.

Como ya se explicó anteriormente, en la actualidad no hay suficientes datos para establecer, con total fundamento, las fórmulas para calcular la composición de fases de los clínkeres, puesto que la desviación respecto a la composición real de aquélla, predecida por las ecuaciones de Bogue y Nurse, indican la necesidad de una fórmula perfeccionada. Sin embargo, se aprecia claramente, a partir de la única característica mineralógica de los clínkeres de Uganda con alto contenido en $\mathrm{Fe}_{2} \mathrm{O}_{3}$, que un simple conjunto de ecuacio- 
nes es improbable sea suficiente para predecir la composición de las fases de todos los clínkeres fosfáticos, particularmente cuando se encuentran presentes cantidades significativas de fluoruros. No son suficientes los datos para poder establecer una nueva fórmula de saturación en cal y de dosificación. Sin embargo la necesidad de tales fórmulas modificadas es menos apremiante, puesto que la usual fórmula de Lea y Parker ha probado ser de utilidad al realizar la dosificación de dos especies diferentes de materias primas. Sin embargo esta simple aplicación de la fórmula es fortuita, puesto que a medida que aumenta el contenido en fosfatos la fórmula de Bogue predice un creciente aumento de las falsas composiciones mineralógicas.

Desde el punto de vista práctico el presente trabajo, realizado con diferentes materias primas fosfáticas ha: 1) confirmado la necesidad de controlar el contenido de $\mathrm{P}_{2} \mathrm{O}_{5}$ del cemento por debajo de un 2,5\% y mantener muy rigurosamente los niveles de fluoruros y $\mathrm{SO}_{3}$; y 2) ha demostrado que el factor de saturación en cal de Lea y Parker puede proporcionar, ocasionalmente, una base práctica para dosificar las materias primas fosfáticas, aunque este factor no establece tolerancias para los efectos del $\mathrm{P}_{2} \mathrm{O}_{5}$ y para el equilibrio de la fase de fluoruros.

\section{AGRADECIMIENTO}

Los autores quieren hacer patente su agradecimiento a los señores G. J. Osborne y J. D. Matthews por la ayuda que les han prestado para la realización de este trabajo de investigación.

\section{R E F E R E N C I A S}

(1) Gutr, W.: Fabricación de cemento portland a partir de materias primas fosfáticas, Proc. 5th International Symposium on the chemistry of Cement, Tokyo 1968, Vol. 1, pp. 93-105. Cement Association of Japan, Tokyo 1970.

(2) Gutr, W y Smith, M. A.: La utilización del fosfogipsum como materia prima en la fabricación del cemento portland. Cement Technology, Vol. 2, 1971: pp. 41-50, y n. ${ }^{\circ} 3$, pp. 91-93 y 100.

(3) Gutr, W. y Nurse, R. W.: Composición de las fases del cemento portland, informe principal. 6th Symposium on the Chemistry of Cement. iMoscú 1974.

(4) NuRse, $\mathrm{R}$ W..: La influencia de los fosfatos sobre la constitución y el endurecimiento del cemento portland. Jour. Applied Chemistry Vol. 2, n. 12, 1952, pp. 708-716.

(5) WeLCH, J. H. y Gutr, W.: El efecto de los componentes secundarios sobre la hidraulicidad de los silicatos cálcicos. Proc. 4th Inter. Symposium on the Chemistry of cement, Washingtom 1960. Vol. 1, pp. 59-68.

(6) Gutr, W.: Equilibrio de fase a alta temperatura en los sistemas de muchos componentes. London University 1966 ( $\mathrm{PhD}$ tesis).

(7) Gutr, W. y Osborne, G. J.: La influencia del potasio sobre la hidraulicidad del silicato bicálcico. Cement Technology, Vol. n. ${ }^{\circ}$ 4, 1970, pp. 121-5.

(8) Midgley, H. G.: El polimorfismo del ortosilicato cálcico. 6th Inter. Symposium on the Chemistry of Cement. Moscú, 1974.

(9) Smith, M. A. y Matthews. J. D.: Estudios con el calorímetro de conducción de los efectos del sulfato sobre las reacciones de hidratación del cemento portland. Cement and Concrete Research, Vol. 4, 1974, pp. 45-55-851.

(10) Gutr, W. y Smith, M. A.: Estudios sobre la misión del sulfato cálcico en la fabricación del cemen. to portland. Trans British Ceram Society, Vol. 67, n. 10 , 1968, pp. 487-509.

(11) Forrester, J. A.: Un calorímetro de conducción para el estudio de la hidratación del cemento. Cement Technology, Vol. 1, n. ${ }^{\circ} 3,1970$, pp. 95-99. 